\title{
MBM 12: young protoplanetary discs at high galactic latitude
}

\author{
G. Meeus ${ }^{1}$, A. Juhász ${ }^{2}$, Th. Henning ${ }^{2}$, J. Bouwman ${ }^{2}$, C. Chen ${ }^{3}$, W. Lawson ${ }^{4}$, D. Apai ${ }^{3}$, \\ I. Pascucci ${ }^{5}$, and A. Sicilia-Aguilar ${ }^{2}$
}

\author{
1 Astrophysical Institute Potsdam, An der Sternwarte 16, 14482 Potsdam, Germany \\ e-mail: gwen@aip.de \\ 2 Max Planck Institute for Astronomy, Königstuhl 17, 69117 Heidelberg, Germany \\ 3 Space Telescope Science Institute, 3700 San Martin Dr., Baltimore, MD 21218, USA \\ 4 School of Physical, Environmental and Mathematical Sciences, University of New South Wales, \\ Australian Defence Force Academy, ACT 2600, Canberra, Australia \\ 5 Department of Physics and Astronomy, John Hopkins University, Baltimore, MD 21218, USA
}

Received 9 December 2008 / Accepted 16 January 2009

\begin{abstract}
We present Spitzer infrared observations to constrain disc and dust evolution in young T Tauri stars in MBM 12, a star-forming cloud at high latitude with an age of $2 \mathrm{Myr}$ and a distance of $275 \mathrm{pc}$. The region contains $12 \mathrm{~T}$ Tauri systems, with primary spectral types between K3 and M6; 5 are weak-line and the rest classical T Tauri stars. We first use MIPS and literature photometry to compile spectral energy distributions for each of the 12 members in MBM 12, and derive their IR excesses. Of the 8 stars that are detected with MIPS (spectral types between K3 and M5), only 1 lacks an IR excess - the other 7 all have an IR excess that can be attributed to a disc. This means that in MBM 12, for the detected spectral types K3-M5, we have a very high disc fraction rate, about $90 \%$. Furthermore, 3 of those 7 excess sources are candidate transitional discs. The four lowest-mass systems in the cloud, with spectral types of M5-M6, were undetected by Spitzer. Their upper limits indicate that they either have a transitional disc, or no disc at all. The IRS spectra are analysed with the newly developed two-layer temperature distribution (TLTD) spectral decomposition method. For the $7 \mathrm{~T}$ Tauri stars with a detected IR excess, we analyse their solid-state features to derive dust properties such as mass-averaged grain size, composition and crystallinity. The mass-averaged grain size we determine from the 10 micron feature has a wide range, between 0.4 and $6 \mu \mathrm{m}$. This grain size is much smaller in the longer-wavelength region: between 0.1 and $1.5 \mu \mathrm{m}$. We find that latertype objects have larger grain sizes, as was already shown by earlier studies. Furthermore, we find a wide range in mass fraction of the crystalline grains, between 3 and (at least) 30\%, with no relation to the spectral type nor grain size. We do find a spatial gradient in the forsterite to enstatite range, with more enstatite present in the warmer regions. The fact that we see a radial dependence of the dust properties indicates that radial mixing is not very efficient in the discs of these young T Tauri stars. The sources that have the least amount of disc flaring have the largest grain sizes, pointing to dust settling. A comparison between the objects with companions closer than 400 AU ("binaries") and those with wider or no companions ("singles"), shows that disc evolution already starts to differentiate at an age of 2 Myr: the excess at $30 \mu \mathrm{m}$ is a factor of 3 greater for the "single" group. The SED analysis shows that the discs in MBM 12, in general, undergo rapid inner disc clearing, while the binary sources have faster disc evolution. The dust grains seem to evolve independently of the stellar properties, but are mildly related to disc properties such as flaring and accretion rates.
\end{abstract}

Key words. circumstellar matter - stars: pre-main sequence - stars: planetary systems: protoplanetary disks - infrared: stars stars: late-type

\section{Introduction}

Our understanding of the star and planet formation process has advanced through comprehensive observations of nearby star-forming regions, such as the Taurus-Auriga cloud or the much denser and more massive Orion Nebula Cluster. Because these sites are relatively close and young, it is often possible to determine the complete census of cluster members, from the highest mass stars down to brown dwarfs with masses of just a few Jupiters (e.g. Luhman et al. 2003; Muench et al. 2002). Furthermore, the detection of companions through adaptive optics and other high-resolution imaging and the subsequent derivation of multiplicity rates is an important piece of the star formation (SF) puzzle (e.g. Petr et al. 1998; Ratzka et al. 2005). In addition, near-IR studies of SF regions have shown that young objects exhibit near-IR excesses, commonly attributed to the presence of protoplanetary discs (e.g. Adams et al. 1987;
Lada et al. 2000). The structure, mass and size of those discs provide constraints on the process of planet formation in these young environments.

To study the young cluster members in more detail, it is important to characterise their circumstellar environment, which is best done at infrared and/or millimetre wavelengths. With the launch of Spitzer, this field has made huge steps forward, as for the first time, it was possible to observe the disc and dust characteristics of large samples of T Tauri stars (e.g. Kessler-Silacci et al. 2006; Sicilia-Aguilar et al. 2007; Pascucci et al. 2008) and even brown dwarfs (Apai et al. 2005; Scholz et al. 2007).

Cloud 12 in the catalogue of Magnani et al. (1985, MBM 12) is a molecular cloud at high galactic latitude $\left(l, b=159.4^{\circ}\right.$, $\left.-34.3^{\circ}\right)$, with relatively high extinction $\left(A_{\mathrm{V}}>5 \mathrm{mag}\right)$. Radio maps of the region in $\mathrm{CO}$ give a mass estimate for the whole complex of 30 to $200 M_{\odot}$ (Pound et al. 1990). The cloud does not appear to be gravitationally bound, and the molecular gas is 
Table 1. Target coordinates and parameters.

\begin{tabular}{|c|c|c|c|c|c|c|c|c|c|}
\hline Object & Alternative name & $\begin{array}{l}\alpha(2000.0) \\
(\mathrm{h} \mathrm{m} \mathrm{s})\end{array}$ & $\begin{array}{c}\delta(2000.0) \\
\left({ }^{\circ},{ }^{\prime} \prime\right)\end{array}$ & $\begin{array}{c}\text { Spectral } \\
\text { type }\end{array}$ & $\begin{array}{l}T_{\text {eff }} \\
(\mathrm{K})\end{array}$ & $\begin{array}{c}\text { Eq. Width }{ }^{a} \\
\mathrm{H} \alpha\end{array}$ & Class $^{b}$ & $\begin{array}{l}\text { Multiplicity } \\
\text { status }\end{array}$ & $\begin{array}{c}\text { Projected }^{c} \\
\text { separation (") }\end{array}$ \\
\hline MBM 12-1 & RX J0255.4+2005 & 025525.78 & 200451.7 & K6 & 4205 & -1 & WTTS & $\bar{B}$ & 0.533 \\
\hline MBM $12-2$ & $\mathrm{LkH} \alpha 262$ & 025607.99 & 200324.3 & M0 & 3850 & -40 & CTTS & $(\mathrm{Q})^{e}$ & 15.3 \\
\hline MBM 12-3 & $\mathrm{LkH} \alpha 263(\mathrm{~A}-\mathrm{B} / \mathrm{B}-\mathrm{C})$ & 025608.42 & 200338.6 & M3 & 3415 & -25 & CTTS & $\mathrm{T}$ & $0.416 / 4.1$ \\
\hline MBM 12-4 & $\mathrm{LkH} \alpha 264$ & 025637.56 & 200537.1 & K5 & 4350 & -18 & CTTS & B & 9.160 \\
\hline MBM 12-5 & E $02553+2018$ & 025811.23 & 203003.5 & K3 & 4660 & -3 & $\mathrm{~W} / \mathrm{CTTS}^{f}$ & B & 1.144 \\
\hline MBM 12-6 & RX J0258.3+1947 & 025816.09 & 194719.6 & M5 & 3200 & -29 & CTTS & $\mathrm{S}$ & - \\
\hline MBM 12-7 & RX J0256.3+2005 & 025617.98 & 200609.9 & M5.75 & 3024 & -14 & WTTS & - & - \\
\hline MBM 12-8 & - & 025749.02 & 203607.8 & M5.5 & 3058 & -120 & CTTS & - & - \\
\hline MBM 12-9 & - & 025813.37 & 200825.0 & M5.75 & 3024 & -10 & WTTS & - & - \\
\hline MBM 12-10 & - & 025821.10 & 203252.7 & M3.25 & 3379 & -12 & WTTS & B & 0.390 \\
\hline MBM 12-11 & - & 025843.80 & 194038.3 & M5.5 & 3058 & -14 & WTTS & - & - \\
\hline MBM 12-12 & S 18 (A-B/Ba-Bb) & 030221.05 & 171034.2 & M3 & 3415 & -69 & CTTS & $\mathrm{T}$ & $0.747 / 0.063$ \\
\hline
\end{tabular}

${ }^{a} \mathrm{H} \alpha$ equivalent width of the primary from Luhman (2001). A negative equivalent width means emission; the width is given in angstroms, A.

${ }^{b}$ T Tauri Class as derived by us (see Sect. 2.1).

${ }^{c}$ From Chauvin et al. (2002) and Brandeker et al. (2003).

${ }^{d}$ S: single, B: binary, T: triple and Q: quadruple.

${ }^{e}$ Possible companion to $\mathrm{LkH} \alpha 263 \mathrm{ABC}$.

${ }^{f}$ On the border between weak-line and classical TTS, see target notes in Sect. 2.1.

expected to dissipate within the next few Myrs (Zimmermann \& Ungerechts 1990). Hearty et al. (2000) used ROSAT observations to detect X-ray sources in MBM 12, and followed up stellar candidates by optical spectroscopy, to confirm 8 X-ray emitting $\mathrm{T}$ Tauri stars with an upper age of $10 \mathrm{Myr}$. It is one of the few clouds at high galactic latitude known to harbour such young objects. From its content, the cloud can be seen as a precursor of a TW Hydrae-like association, where the molecular material has not yet disappeared. Although MBM 12 was initially thought to be one of the nearest star formation regions, at a distance of only $65 \mathrm{pc}$, it is now determined to lie at a distance of $275 \mathrm{pc}$ (Luhman 2001).

A census of this association - complete down to masses of $0.03 M_{\odot}-$ was determined by Luhman (2001), based on sensitive near-IR and optical photometry. The candidate members were confirmed by follow-up spectroscopy, which also allowed them to study their $\mathrm{H} \alpha$ and $\mathrm{Li} 6707 \AA$ line properties. In total, $12 \mathrm{~T}$ Tauri stars were found to be real members of MBM 12, and an age of $2_{-1}^{+3}$ Myr was derived from both the lithium line and the location of the objects in the H-R diagram. The spectral types for those 12 TTS is between K3 and M6. An additional study by Luhman \& Steeghs (2004) of 7 candidate members could not confirm any more members. The T Tauri stars in MBM 12 have a high binary frequency: near-infrared adaptive optics studies by Chauvin et al. (2002) and Brandeker et al. (2003) revealed that MBM 12 contains at least 4 binaries and 2 triples with projected separations between 20 and $4000 \mathrm{AU}$ (of which one is a candidate quadruple: $\mathrm{LkH} \alpha 262$ has a projected distance of only 15 arcsec to the triple $\mathrm{LkH} \alpha 263$ ).

In this paper, we present Spitzer IRS and MIPS observations of all MBM 12 members, and combine our observations with literature photometry and spectroscopy to analyse the derived dust characteristics by relating them to the stellar parameters and disc properties. In Sect. 2, we present the individual targets and the Spitzer observations. The analysis in Sect. 3 first discusses the spectral energy distributions, and then the spectral features are interpreted in terms of dust properties with the aid of a spectral decomposition model. In Sect. 4, we discuss grain growth, crystallisation, disc properties and accretion rates. We round off with conclusions in Sect. 5, and give more details in the Appendix on the dust model used and the mass fractions derived.

\section{Sample and observations}

\subsection{Targets}

Our sample is unbiased, as it includes all the confirmed members of the MBM 12 cloud (Luhman 2001, 2004). Their parameters, spectral type and T Tauri Class (weak-line or classical) are listed in Table 1. We re-derived their T Tauri Class, following White and Basri (2003): T Tauri stars are classical when, for spectral types between $\mathrm{K} 0$ and $\mathrm{K} 5$, the equivalent width of their $\mathrm{H} \alpha$ line, $|E W(\mathrm{H} \alpha)| \geq 3 \AA$, for $\mathrm{K} 7$ to $\mathrm{M} 2.5,|E W(\mathrm{H} \alpha)| \geq 10 \AA$ and for M3 to M5.5, $|E W(\mathrm{H} \alpha)| \geq 20 \AA$. We also list their multiplicity status and projected separation of companions (when present), as determined by Brandeker et al. (2003) and Chauvin et al. (2002) for 8 of the 12 members. The objects under consideration are all T Tauri stars with spectral types between K3 and M5.75, and at least 6 out of the 12 targets are known to have companions. Below, we list more information on the individual targets:

- MBM 12-1 (RX J0255.4+2005) is a weak-line binary, with the primary of spectral type K6 and a separation of 0.533 (Chauvin et al. 2002). X-ray observations with ROSAT showed that this object flares, with a factor of six increase in X-ray counts during the flare. Spectral fits of the count rate suggest a coronal origin, not untypical of other flaring WTTS (Hearty et al. 2000).

- $\mathbf{L k H} \alpha \mathbf{2 6 2}$ is a CTTS, with a disc detected at $2.1 \mathrm{~mm}$, from which Itoh et al. (2003) estimated a disc mass of $0.05 M_{\odot}$. It is located at a distance of only $15^{\prime \prime} .3$ from $\mathrm{LkH} \alpha 263$.

- LkH $\alpha 263$ is a triple system, with $\mathrm{LkH} \alpha 262$ possibly belonging to this triple to then form a quadruple system (Chauvin et al. 2002); however, it is not clear whether this system is bound. The C component of $\mathrm{LkH} \alpha 263$ has spectral type M0, and was found to harbour an optically thick disc, that spatially-resolved observations showed to be edgeon (Jayawardhana et al. 2002). These authors derived a disc mass of $0.0018 M_{\odot}$ for this $0.7 M_{\odot} \mathrm{C}$ component, based on their near-IR images. Furthermore, forbidden lines in the optical spectrum of $\mathrm{LkH} \alpha 263 \mathrm{C}$ suggest the presence of a jet (Jayawardhana et al. 2002).

- $\mathbf{L k H} \alpha \mathbf{2 6 4}$ is a wide binary with a separation of 9.'160. Millimetre observations at 1.3 and $2.1 \mathrm{~mm}$ give a disc mass 
of $0.09 M_{\odot}$ around the primary (Itoh et al. 2003). Emission of molecular hydrogen at 2.1218 and $2.2233 \mu \mathrm{m}$ was also detected around the primary. The width of these lines points to a disc origin, while further modelling locates the NIR emitting $\mathrm{H}_{2}$ in the inner $10 \mathrm{AU}$, and shows that the disc is seen nearly pole-on (Carmona et al. 2008). The line strength ratio is consistent with a temperature lower than $1500 \mathrm{~K}$, and points to thermal excitation by UV photons; $\mathrm{LkH} \alpha 264$ has a strong UV excess, so it is indeed plausible that there are enough UV photons to excite the $\mathrm{H}_{2}$. The total mass of the optically thin, hot $\mathrm{H}_{2}$ in the disc of the primary is estimated to be a few lunar masses (Carmona et al. 2008).

- MBM 12-5 (E 0255+2018) is a binary TTS with a separation of 1". 144. Following the classification by White \& Basri (2003), its spectral type of $\mathrm{K} 3$ and $\mathrm{H} \alpha$ equivalent width of $3.1 \AA$ puts it on the border between the classical and weakline $\mathrm{T}$ Tauri stars.

- MBM 12-6 is a classical T Tauri star for which Brandeker et al. (2003) did not find a companion within a radius of 1". 6 .

- MBM 12-7, 9 and 11 are all weak-line T Tauri stars, with the latest spectral types of the whole MBM 12 sample: between M5 and M6, corresponding to masses between 0.15 and $0.1 M_{\odot}$. We could not find any multiplicity data for these relatively faint objects. High spatial resolution images are needed to establish the multiplicity status of these sources.

- MBM 12-8 is a classical TTS, with spectral type M5.5, and the highest $\mathrm{H} \alpha$ equivalent width: $120 \AA$, suggesting it is the most actively accreting object of the sample. No additional information concerning its multiplicity status is available.

- MBM 12-10 is a binary weak-line TTS with a separation of 0.390 .

- S 18 is a triple system, consisting of the primary A and, at a projected distance of 0.747 , a tight binary companion Bab (with a separation of only 0.'063 - Brandeker et al. 2003). Millimetre observations at $2.1 \mathrm{~mm}$ give a disc mass of $0.07 M_{\odot}$ around the primary (Itoh et al. 2003).

\subsection{Spitzer IRS spectroscopy}

The T Tauri stars in MBM 12 were observed with Spitzer as part of a larger programme on young stellar clusters to study the evolution of protoplanetary discs (GO proposal 20691, PI Bouwman). We obtained 7.5-35 $\mu \mathrm{m}$ low-resolution $(R=$ 60-120) spectra of the MBM 12 cluster members with the Infrared Spectrograph (IRS, Houck et al. 2004) on-board the Spitzer Space Telescope. A high accuracy PCRS peak-up was executed prior to the spectroscopic observations to position the target within the slit. All targets were observed with a minimum of three observing cycles for redundancy. Our spectra are based on the droopres products processed through the S15.3.0 version of the Spitzer data pipeline. Partially based on the SMART software package (Higdon et al. 2004), our data were further processed using spectral extraction tools developed by the "Formation and Evolution of Planetary Systems" (FEPS) Spitzer science legacy team (see also Bouwman et al. 2008). The spectra were extracted using a 6.0 pixel and 5.0 pixel fixed-width aperture in the spatial dimension for the observations with the first order of the short- (7.5-14 $\mu \mathrm{m})$ and the long-wavelength (14-35 $\mu \mathrm{m})$ modules, respectively. The background was subtracted using associated pairs of imaged spectra from the two nodded positions along the slit, also eliminating stray light contamination and anomalous dark currents. Pixels flagged by the data pipeline as being "bad" were replaced with a value interpolated from an 8 pixel perimeter surrounding the errant pixel. The low-level fringing at wavelengths $>20 \mu \mathrm{m}$ was removed using the irsfringe package (Lahuis \& Boogert 2003). To remove any effect of pointing offsets perpendicular to the slit, we matched orders based on the point spread function of the IRS instrument, correcting for possible flux losses (see Swain et al. 2008, for further details). To remove any effect of pointing offsets parallel to the slit, we made a flatfield correction to the nominal rsrf which was derived from calibration measurements of standard stars where the calibrator was mapped along the slit. The spectra are calibrated using a spectral response function derived from multiple IRS spectra of the calibration star $\eta_{1}$ Doradus and a MARCS stellar model provided by the Spitzer Science Centre. The spectra of the calibration target were extracted in the same way as our science targets. The relative errors between spectral points within one order are dominated by the noise on each individual point and not by the calibration. We estimate a relative flux calibration across an order of $\approx 1 \%$ and an absolute calibration error between orders/modules of $\approx 3 \%$, which is mainly due to uncertainties in the scaling of the MARCS model.

The targets with the latest spectral types, MBM 12-7, 8, 9 and 11, were not detected with IRS, so we will not include them in the rest of our analysis of the dust properties. This means that the spectral range for which we have IR spectroscopy is limited to K3-M5.

\subsection{Spitzer MIPS photometry}

Additional infrared data was obtained using MIPS (Rieke et al. 2004) on Spitzer (Werner et al. 2004) in photometry mode at 24 and $70 \mu \mathrm{m}$ (default scale). All of our targets were observed in February 2006, using 1 cycle of $3 \mathrm{sec}$ integrations at $24 \mu \mathrm{m}$ and $2-5$ cycles of $10 \mathrm{~s}$ integrations at $70 \mu \mathrm{m}$, corresponding to on-source intergation times of $24.1 \mathrm{~s}$ and 251.6-629 s at 24 and $70 \mu \mathrm{m}$, respectively. Our observations were processed using version S16.0.1 of the Spitzer Science Center (SSC) data pipeline. We created two $24 \mu \mathrm{m}$ mosaics for each of our sources from the resulting basic calibrated data (BCD) images of each data collection event (DCE) using the SSC's MOPEX software (Makovitz \& Marleau 2005), one with a pixel scale approximately that of the native pixel scale $\left(2{ }^{\prime \prime} 45\right.$ pixel $\left.^{-1}\right)$ and another resampled to approximately one half of the native pixel scale $\left(1^{\prime \prime} .23\right.$ pixel $\left.^{-1}\right)$. We similarly created two $70 \mu \mathrm{m}$ mosaics for each of our sources using the filtered BCDs (which have spatial and temporal filters applied to the data in order to remove instrumental signatures) with outlier rejection, one with a pixel approximately that of the native pixel scale $\left(9.9\right.$ pixel $\left.^{-1}\right)$ and another resampled to approximately one quarter of the native pixel scale $\left(2\right.$ '. $^{\prime}$ pixel $\left.^{-1}\right)$.

We used the APEX portion of MOPEX to perform aperture photometry on our mosaicked images. APEX applies a median filter to the data to estimate the sky background at any pixel in the image and subtracts the median-filtered image before summing the flux in an aperture. Since the estimated backgrounds in the majority of our fields, extrapolated from COBE (Cosmic Microwave Background Explorer), was medium to high, we used a medium-sized aperture with radius of $6^{\prime \prime}$ at $24 \mu \mathrm{m}$ and $16^{\prime \prime}$ at $70 \mu \mathrm{m}$ to reduce the amount of background contamination in the aperture. These apertures are not large enough to contain all of the photons from a diffraction-limited point source; therefore, we applied scalar aperture corrections of 1.697 and 1.771 at $24 \mu \mathrm{m}$ and $70 \mu \mathrm{m}$, as published on the SSC website. The $70 \mu \mathrm{m}$ aperture correction is somewhat dependent on the colour of the source; the aperture correction used here assumes that 
Table 2. MIPS photometry and the statistical errors.

\begin{tabular}{ccc}
\hline Object & $\begin{array}{c}24 \mu \mathrm{m}(1 \sigma) \\
(\mathrm{mJy})\end{array}$ & $\begin{array}{c}70 \mu \mathrm{m}(1 \sigma) \\
(\mathrm{mJy})\end{array}$ \\
\cline { 2 - 3 } MBM 12-1 & $2.8(0.4)$ & $<34.4$ \\
MBM 12-2 & $142(0.4)$ & $216^{a}(12)$ \\
MBM 12-3 & $50.8(0.4)$ & $216^{a}(12)$ \\
MBM 12-4 & $282(0.5)$ & $266(13)$ \\
MBM 12-5 & $308(0.5)$ & $253(12)$ \\
MBM 12-6 & $25.7(0.4)$ & $<34.4$ \\
MBM 12-7 & $<1.2$ & $<32.9$ \\
MBM 12-8 & $<1.2$ & $<41.4$ \\
MBM 12-9 & $<1.2$ & $<37.5$ \\
MBM 12-10 & $21(0.4)$ & $<35.6$ \\
MBM 12-11 & $<1.2$ & $<34.1$ \\
MBM 12-12 & $45.5(0.4)$ & $<32.2$ \\
\hline
\end{tabular}

the measured flux has a red power law shape, $F_{v} \propto v^{-2}$, because any flux detected at $70 \mu \mathrm{m}$ is dominated by the emission from a cool, dusty disc $(T \sim 100 \mathrm{~K})$. The MIPS data handbook (version 3.2.1) states that products processed with version S14.4 of the data pipeline have absolute calibration uncertainties of 4 and $7 \%$ at 24 and $70 \mu \mathrm{m}$, respectively.

The majority of our sources were detected with signal to noise ratios greater than 10 at $24 \mu \mathrm{m}$ and were not detected at $70 \mu \mathrm{m}$ (see Table 2). We estimate $1 \sigma$ flux uncertainties for objects that were detected and $3 \sigma$ upper limits on the fluxes of objects that were not detected from uncertainty mosaics produced by MOPEX (at the native pixel resolution). To determine the $1 \sigma$ uncertainty in a measured flux, we take the square root of the sum of the uncertainties (in the flux in each pixel in the aperture) in quadrature, multiplied by the aperture correction (1.164 and 1.197 at 24 or $70 \mu \mathrm{m}$, respectively), centered at the expected position of the source. Similarly, we determine the $3 \sigma$ upper limit on the 24 and $70 \mu \mathrm{m}$ fluxes as three times the square root of the sum of uncertainties (in the flux in each pixel in the aperture) in quadrature, multiplied by the aperture correction (1.164 and 1.197 , respectively), in a relatively large aperture, with radius $35^{\prime \prime}$, centered at the expected position of the source.

In Table 2, we list the MIPS photometry for the MBM 12 members. The 4 stars with the latest spectra types, MBM 12-7, 8,9 and 11 were not detected at 24 nor at $70 \mu \mathrm{m}$. In Fig. 2, we show that the MIPS photometry at $24 \mu \mathrm{m}$ and the fluxes from the IRS spectra agree very well.

\section{Analysis}

\subsection{Spectral energy distributions}

To complement our Spitzer observations, we collected photometry from the literature: Jayawardhana et al. (2001) and Luhman (2001) for $R I, J H K_{\mathrm{s}}$ and $L N$ band photometry. The $V$ band is from Broeg et al. (2006), while the submillimetre and millimetre photometry are from Hogerheijde et al. (2003) and Itoh et al. (2003), respectively. In Fig. 2, we show the spectral energy distributions (SED) of the 12 targets, together with a MARCS stellar model (Gustafsson et al. 2008) and for those sources with $T_{\text {eff }}>4200 \mathrm{~K}$ with a Kurucz atmosphere model (Kurucz 1994) for the appropriate effective temperature of the central source, as listed in Table 1 . The photometry was dereddened using the $A_{V}$ values derived by Luhman (2001), assuming a standard extinction law $\left(R_{V}=3.1\right)$.

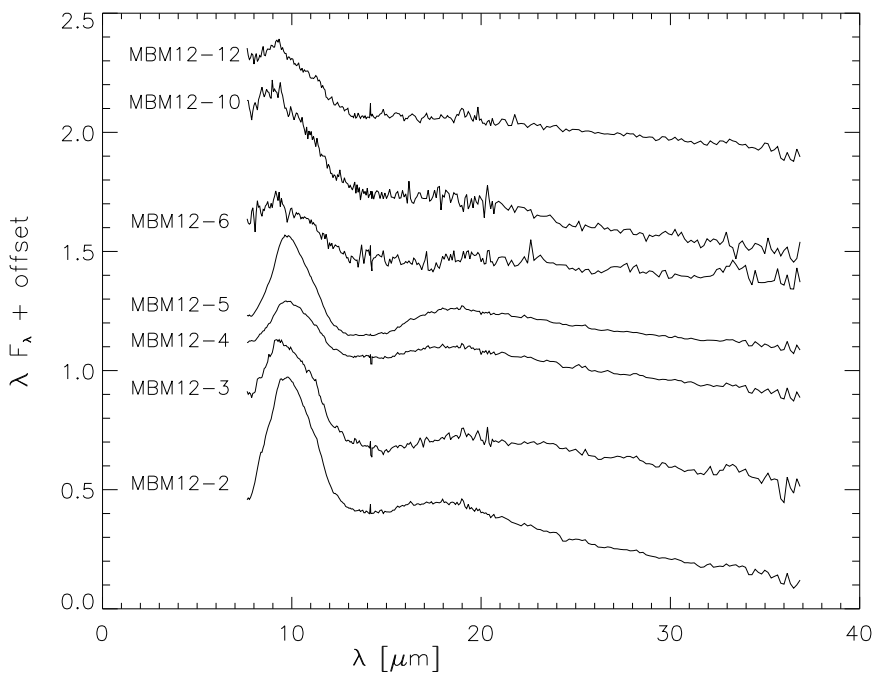

Fig. 1. IRS Spitzer spectra of the seven MBM 12 sources that show excess emission in the infrared. The strength and shape of the emission features, as well the slope of the spectra varies from source to source.

Of the 8 sources detected with Spitzer, only one object, MBM 12-1, a K6-type weak-line TTS with a companion at 0.533 , shows no excess emission at all: the spectrum is purely atmospheric. The other 7 sources show an infrared excess, and also have a silicate emission feature at $10 \mu \mathrm{m}$, with varying degrees of strengths and shapes (see Fig. 1). As we do not have any data between $\sim 3.4$ and $8 \mu \mathrm{m}$, it is difficult to determine the properties of the inner disc, e.g. whether there is an inner hole or not, as witnessed by a lack of excess shortwards of the $10 \mathrm{mi}-$ cron feature. Sources MBM 12-3, 6 and 10, appear not to have a near-IR excess (see Fig. 2); they are candidate transitional discs, where the inner disc has already been cleared out. MBM 12-2, 4, 5 and 12 have "full discs", as they already have an excess in the $K$ band. Additional data, between 3 and 8 micron, is needed to confirm the status of the transitional candidates. An overview of the main features observed is given in Table 3; the properties of the dust, as derived from the solid-state features, will be discussed in the following section. We discuss the relation between excess luminosity and binarity in Sect. 4.3.

\subsection{Dust mineralogy}

The most frequently analysed region in the context of dust properties is the 10 micron region: it is through this window that dust characteristics like composition (e.g. olivines, $\mathrm{Mg}_{2 \mathrm{x}} \mathrm{Fe}_{2(1-\mathrm{x})} \mathrm{SiO}_{4}$ and pyroxenes, $\mathrm{Mg}_{\mathrm{x}} \mathrm{Fe}_{(1-\mathrm{x})} \mathrm{SiO}_{3}$, or $\mathrm{Mg} / \mathrm{Fe}$ content), structure (crystalline vs. amorphous) and grain sizes can be derived from their solid-state features. The $10 \mu \mathrm{m}$ region is only sensitive to dust grains up to sizes of a few microns, as the feature flattens out when the dust grains attain sizes similar to the wavelength, making them essentially invisible. Furthermore, it is important to realise that the dust causing this feature traces only a small fraction of the disc material, namely those dust grains that are located in the optically thin disc atmosphere, while the bulk of the dust mass is located in the disc midplane. In addition, as the dust temperature needs to be high enough (150-450 K) in order to radiate at $10 \mu \mathrm{m}$, the radial location of the dust observed is also limited: for a typical star in our sample, this is around $1 \mathrm{AU}$. At longer wavelengths, features of crystalline dust (forsterite and enstatite) reflect another important part of dust processing in the protoplanetary disc. 


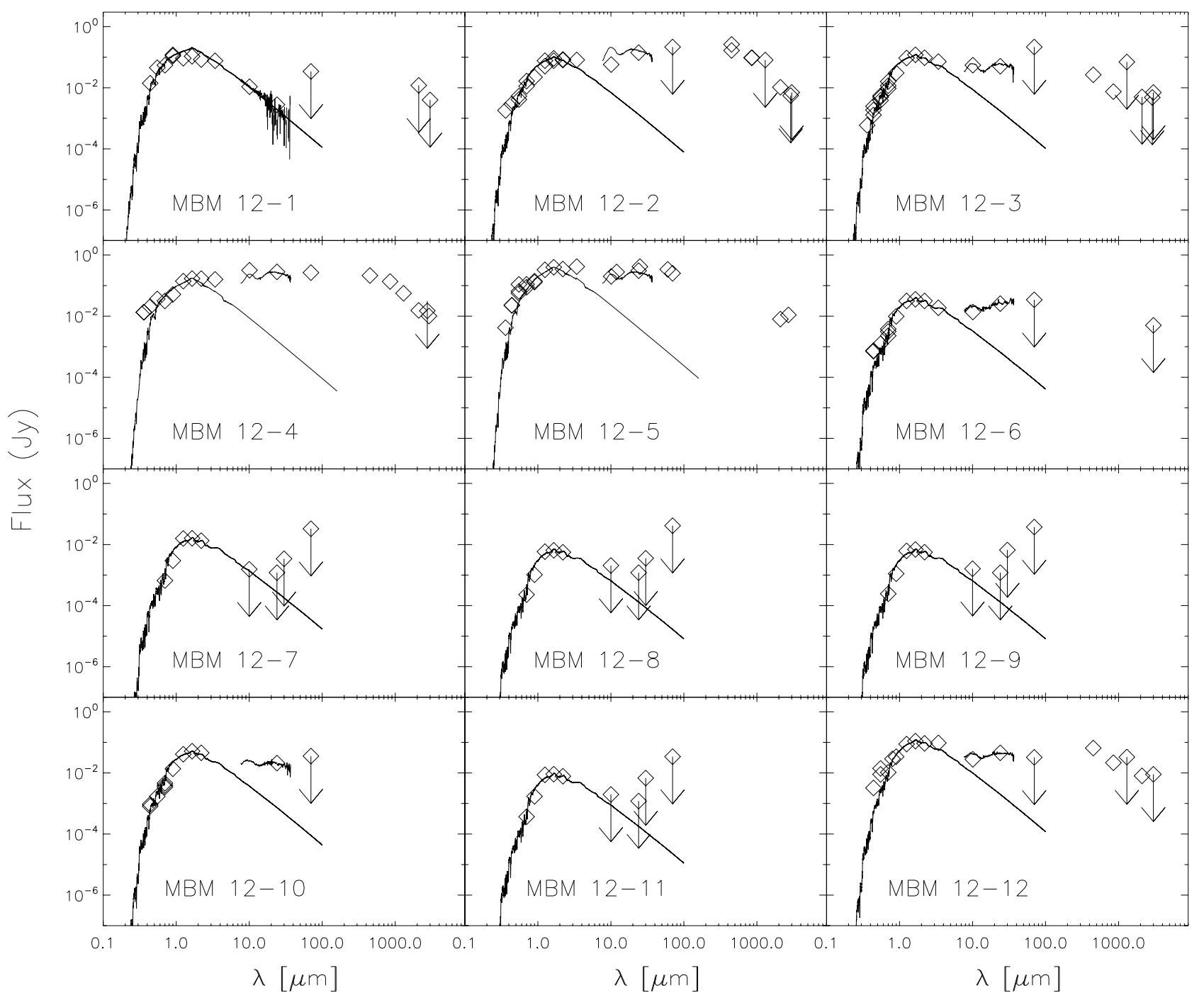

Fig. 2. The spectral energy distributions of the $12 \mathrm{~T}$ Tauri stars in MBM 12. The lowest-mass stars, MBM 12-7, 8, 9 and 11 were not detected with Spitzer, therefore we can only show upper limits in the infrared for those sources. MBM 12-1 is the only source in our detected sample that shows no excess above its atmosphere. MBM 12-3, 6 and 10 appear to not have a near-IR excess, so are candidate transitional discs, while MBM 12-2, 4, 5 and 12 have "normal accretion discs", with an excess extending into the near-IR.

Table 3. Summary of the spectral appearance of the detected targets.

\begin{tabular}{lccc}
\hline \hline Object & SED $^{a}$ & Enstatite $^{b}$ & Forsterite $^{b}$ \\
\hline MBM 12-1 & Photosphere & - & - \\
MBM 12-2 & Full & - & $19,27.8,33 \mu \mathrm{m}$ \\
MBM 12-3 & TO candidate & $9.3:$ & $11.3:, 19:, 27.8,33 \mu \mathrm{m}$ \\
MBM 12-4 & Full & - & $11.3:, 19:, 27.8,33 \mu \mathrm{m}$ \\
MBM 12-5 & Full & - & $19,27.8,33 \mu \mathrm{m}$ \\
MBM 12-6 & TO candidate & $9.3 \mu \mathrm{m}$ & $27.8,33 \mu \mathrm{m}$ \\
MBM 12-10 & TO candidate & $9.3 \mu \mathrm{m}$ & $23.4,27.8 \mu \mathrm{m}$ \\
MBM 12-12 & Full & $9.3 \mu \mathrm{m}$ & $11.3,19,27.8,33 \mu \mathrm{m}$ \\
\hline
\end{tabular}

a We distinguish between a "full" and a "transitional" disc, based on the presence or absence of excess emission at wavelengths shorter than 8 micron, and write "transitional object (TO) candidate" when this classification needs to be confirmed by additional photometry.

${ }^{b}$ We list the wavelengths at which features of enstatite and forsterite are present. Colons indicate uncertain detections.

In Fig. 3, we zoom in on the three most interesting ranges that are observed with IRS, and also indicate the location of the most important features. Apart from the broad feature of amorphous silicate at 9.7 (present in all 7 stars with a detected excess) and $18 \mu \mathrm{m}$, features of enstatite $\left(\mathrm{MgSiO}_{3}\right)$ and forsterite $\left(\mathrm{Mg}_{2} \mathrm{SiO}_{4}\right)$ are observed. We do not see evidence for carbonaceous dust, such as features from polycyclic aromatic hydrocarbons (PAHs). This is perhaps not surprising, given that PAHs are transiently excited by UV photons and the central sources have low temperatures, although some sources do show a rather large UV excess (e.g. MBM 12-2, 4 and 12).

In order to analyse the composition of the dust in the disc atmosphere, the radiation of which dominates the IRS spectrum, we use the two-layer temperature distribution (TLTD) spectral decomposition routines described in Juhász et al. (2009). This method uses a multi-component continuum (star, inner rim, disc midplane), assuming that the region where the observed radiation originates (both optically thin and thick) has a distribution of temperatures. In this fitting method, the observed flux-density at a given frequency is given by

$F_{v}=F_{v, \text { cont }}+\sum_{i=1}^{N} \sum_{j=1}^{M} D_{i, j} K_{i, j} \int_{T_{a, \text { in }}}^{T_{a, \text { out }}} \frac{2 \pi}{d^{2}} B_{v}(T) T^{\frac{2-q a}{q a}} \mathrm{~d} T$ 

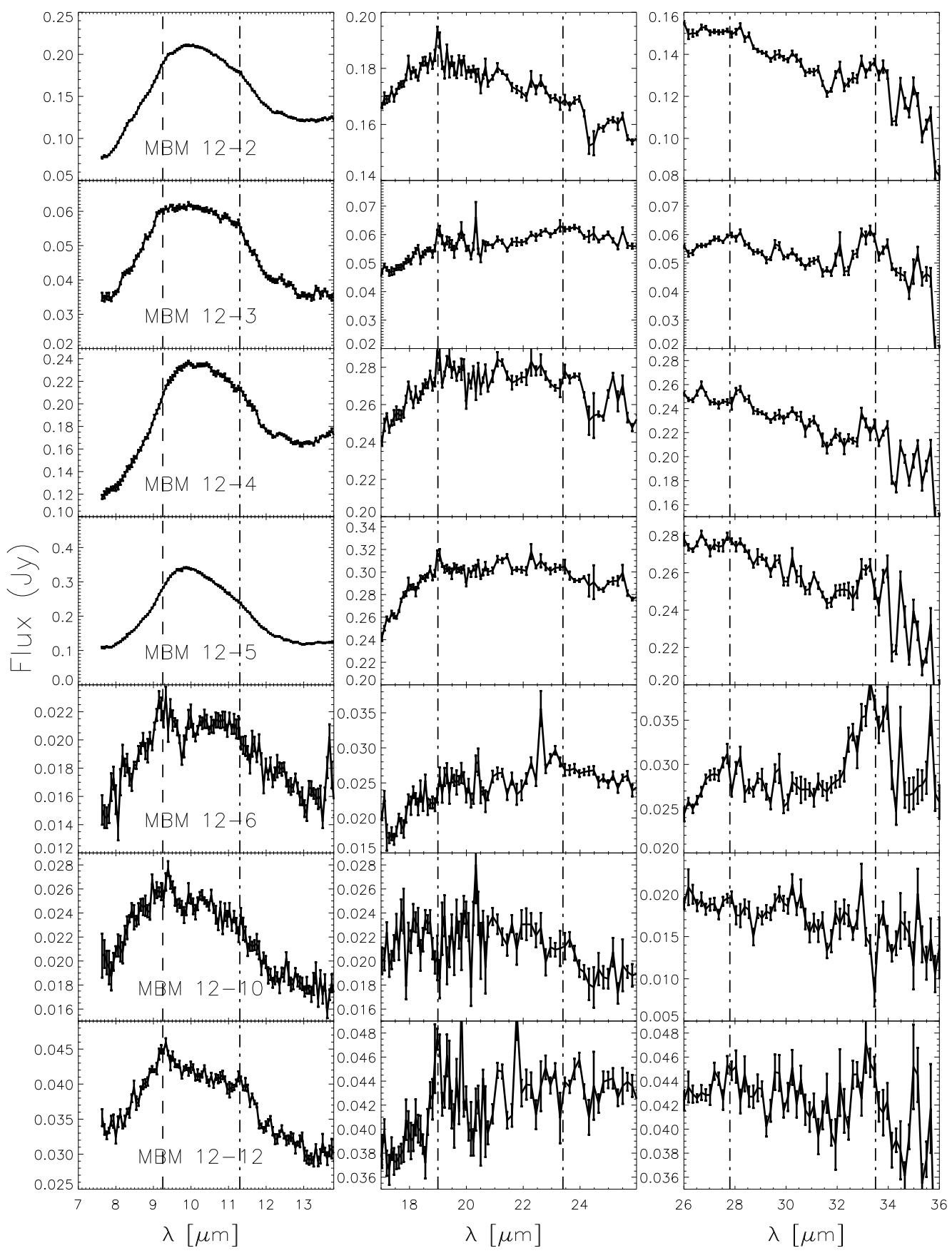

Fig. 3. Zoom in on the 3 main ranges in the IRS spectra: 7-14 $\mu \mathrm{m}, 17-25 \mu \mathrm{m}$ and $26-36 \mu \mathrm{m}$. We overplot the positions of the main crystalline features: long-dashed lines indicate enstatite, while dot-dashed lines indicate forsterite.

where $N$ and $M$ are the number of dust species and of grain sizes used, respectively. $\kappa_{i, j}$ is the mass absorption coefficient of the dust species $i$ and grain size $j . B_{v}(T)$ is the Planck-function, $q a$ is the power exponent of the temperature distribution and $d$ is the distance to the source. The subscript " $a$ " in the integration boundaries refers to the disc atmosphere. The continuum emission $\left(F_{v, \text { cont }}\right)$ is given by

$$
\begin{aligned}
F_{v, \text { cont }}= & D_{0} \frac{\pi R_{\star}^{2}}{d^{2}} B_{v}\left(T_{\star}\right)+D 1 \int_{T_{r, \text { in }}}^{T_{r \text { out }}} \frac{2 \pi}{d^{2}} B_{v}(T) T^{\frac{2-q r}{q r}} \mathrm{~d} T \\
& +D 2 \int_{T_{m, \text { in }}}^{T_{m, \text { out }}} \frac{2 \pi}{d^{2}} B_{v}(T) T^{\frac{2-q m}{q m}} \mathrm{~d} T .
\end{aligned}
$$

The first term on the right hand side describes the emission of the star, while the second and third term describe the radiation of the inner rim (subscript " $r$ ") and the disc midplane (subscript " $m$ "), respectively. The meaning of the different parameters are summarised in Table 7 in the Appendix. For each component (disc atmosphere, inner rim, midplane), the highest temperature is fitted to obtain $T_{a / r / m}$, in, while the lowest temperature, $T_{a / r / m \text {, out }}$, is calculated requiring that the annulus with that temperature contributes more than $0.1 \%$ to the total flux.

For our fit, we used five dust species that are commonly found in the discs of young stars, in three grain sizes $(0.1,1.5$ and $6 \mu \mathrm{m}$ ). To derive the mass absorption coefficients from the optical constants, we applied the theory of distribution of hollow spheres for the crystalline dust to simulate grain shapes 

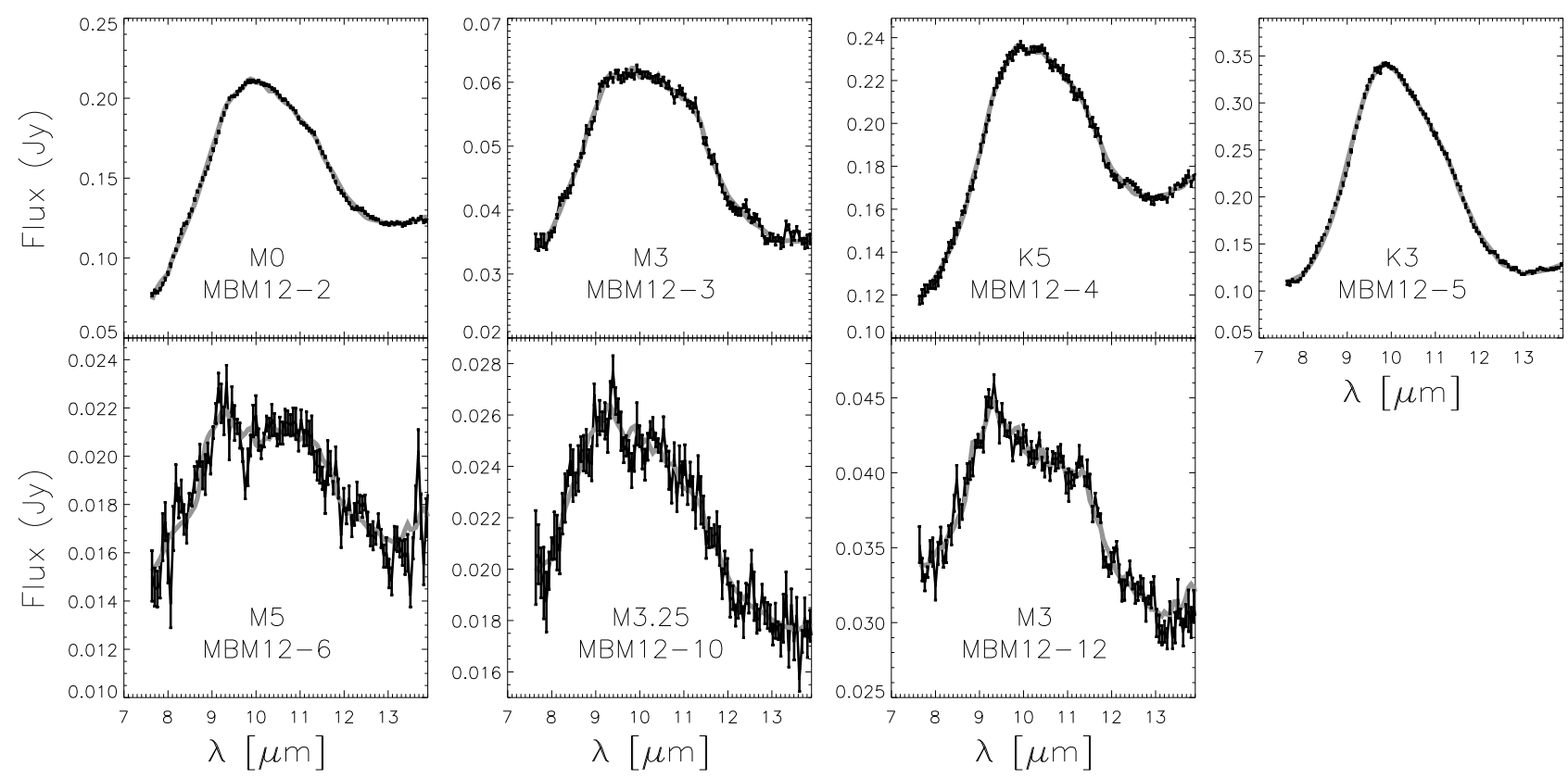

Fig. 4. The TLTD fits of the 10 micron silicate feature (grey, solid line), and the IRS spectra with their noise (black points with error bars). Notice the different shapes that are present in this small sample: MBM 12-5 has a triangular shape, pointing to small, amorphous silicate grains, while MBM 12-6 has a much broader feature, indicating larger and crystalline grains. The narrow emission feature at $9.3 \mu \mathrm{m}$ is caused by crystalline enstatite.

Table 4. Overview of dust species used in our fitting routines.

\begin{tabular}{llll}
\hline \hline Species & State $^{a}$ & $\begin{array}{l}\text { Chemical } \\
\text { formula }\end{array}$ & Shape \\
\hline $\begin{array}{l}\text { Amorphous silicate } \\
\text { (Olivine stoichiometry) }\end{array}$ & $\mathrm{A}$ & $\mathrm{MgFeSiO}_{4}$ & $\begin{array}{l}\text { Homogeneous } \\
\text { Sphere }\end{array}$ \\
$\begin{array}{l}\text { Amorphous silicate } \\
\text { (Pyroxene stoichiometry) }\end{array}$ & $\mathrm{A}$ & $\mathrm{MgFeSi}_{2} \mathrm{O}_{6}$ & $\begin{array}{l}\text { Homogeneous } \\
\text { Sphere }\end{array}$ \\
Forsterite $^{2}$ & $\mathrm{C}$ & $\mathrm{Mg}_{2} \mathrm{SiO}_{4}$ & $\begin{array}{l}\text { Hollow Sphere } \\
\text { Clino Enstatite }^{3}\end{array}$ \\
Silica $^{4}$ & $\mathrm{C}$ & $\mathrm{MgSiO}_{3}$ & Hollow Sphere \\
$\mathrm{SiO}_{2}$ & Hollow Sphere \\
\hline
\end{tabular}

References: (1) Dorschner et al. (1995); (2) Servoin et al. (1973);

(3) Jäger et al. (1998); (4) Henning et al. (1997).

${ }^{a}$ Lattice structure: Amorphous (A) or Crystalline (C).

deviating from perfect symmetry (Min et al. 2005) and the classical Mie theory for spherical particles for the amorphous dust grains. The list of the dust species, the origin of the optical constants and the grain model used are presented in Table 4. Furthermore, since the dust grains radiating at $8-13 \mu \mathrm{m}$ and 20-30 $\mu \mathrm{m}$ are at different temperatures, hence at different radial distances from the star, we split the wavelength region in two parts before fitting. This way, we can take into account that different species can contribute in different amounts to the spectrum, if there is a radial gradient in their abundances and/or properties. We chose the 1) the $7-17 \mu \mathrm{m}$ range for the shorter wavelength region, which Juhász et al. (2009) showed to be the optimal wavelength range to balance the presence of spectral features and radial changes; and 2) the 17-37 $\mu \mathrm{m}$ range for the longer wavelength region, which includes important crystalline features; both regions were separatedly fitted, and we will refer to them as the "shorter" and "longer" wavelength regions.
As the IRS spectra do contain noise, we repeated the fit of each object 100 times, every time adding random Gaussian noise on top of the measured spectrum. The final composition is derived as the average of those 100 fits, while the errors are derived from the standard deviation, taking into account the positive and negative directions of the variations. Therefore, the errors we give describe the $\mathrm{S} / \mathrm{N}$ and measurement errors in the spectra. We emphasize that we cannot give error estimates that are related to species that are not included in our fits, nor of different shapes or composition of the grains. Those errors are the subject of another study (Juhász et al. 2009).

With the fits, we determine the source of the solid-state features that are observed in the spectra. Since several species (e.g. carbon) are featureless within our wavelength range (but can contribute to the continuum), they remain undetected in our fits; so it is important to realise that we can only discuss the "visible dust", that has features in the range 7 to 37 micron. In Fig. 4, we show the best fit of the 7 to 17 micron region, and a summary of the derived dust properties for both the shorter and longer wavelength regions is given in Table 5. The detailed results of the fits in both regions, in terms of mass fractions of the relevant species and their sizes, are given in Table 8 in the Appendix.

\section{Discussion: 2 Myr-old T Tauri discs}

\subsection{Grain growth}

A first thing to notice, when looking at the 10 micron silicate feature, is that the strength of the emission varies. Van Boekel et al. (2003) showed that the shape and the strength of the $10 \mu \mathrm{m}$ feature in Herbig Ae/Be stars are related, and demonstrated it to be evidence of grain growth: a strong and triangular $10 \mu \mathrm{m}$ feature is typical of small (submicron-sized) grains, whereas a weaker and broader feature indicates the presence of larger sized grains (up to a few microns). In Fig. 5, we probed this relation 
Table 5. Distilled fitting results.

\begin{tabular}{|c|c|c|c|c|}
\hline Object & $\begin{array}{c}\langle a\rangle_{\mathrm{am} . \text { sil. }^{a}}{ }^{a} \\
(\mu \mathrm{m})\end{array}$ & $\begin{array}{c}\langle a\rangle_{\text {cryst.sil. }}{ }^{a} \\
(\mu \mathrm{m})\end{array}$ & $\begin{array}{l}f_{\text {cryst. }}^{b} \\
(\%)\end{array}$ & Forst/enst ${ }^{c}$ \\
\hline \multicolumn{5}{|c|}{$7-17 \mu \mathrm{m}$ region } \\
\hline MBM 12-2 & $4.1\left({ }_{-0.1}^{+0.1}\right)$ & $5.2\left({ }_{-0.1}^{+0.1}\right)$ & $10.7\left({ }_{-0.8}^{+0.7}\right)$ & $0.1\left(\begin{array}{c}+0.0 \\
-0.0\end{array}\right)$ \\
\hline MBM 12-3 & $4.0\left(\left(_{-0.6}^{+0.4}\right)\right.$ & $1.7\left(\left(_{-1.1}^{+1.7}\right)\right.$ & $5.8\left({ }_{-1.6}^{+2.1}\right)$ & $0.4\left(\begin{array}{l}+0.1 \\
-0.2\end{array}\right)$ \\
\hline MBM 12-4 & $0.4\left(\begin{array}{l}+0.1 \\
-0.1\end{array}\right)$ & $4.1\left(\begin{array}{c}+0.5 \\
-0.6\end{array}\right)$ & $16.3\left(\begin{array}{l}+2.4 \\
-2.6\end{array}\right)$ & $0.1\left(\begin{array}{c}+0.0 \\
-0.0\end{array}\right)$ \\
\hline MBM 12-5 & $2.2\left(\begin{array}{c}+0.1 \\
-0.0\end{array}\right)$ & $4.1\left(\begin{array}{l}+1.0 \\
-1.6\end{array}\right)$ & $2.9\left(\begin{array}{l}+1.2 \\
-1.1\end{array}\right)$ & $0.3\left(\begin{array}{c}+0.3 \\
-0.1\end{array}\right)$ \\
\hline MBM 12-6 & $3.9\left(\begin{array}{c}+2.0 \\
-3.1\end{array}\right)$ & $5.3\left(\left(_{-0.2}^{+0.0}\right)\right.$ & $46.5\left(\begin{array}{l}+24.9 \\
-16.6\end{array}\right)$ & $0.0\left(\begin{array}{c}+0.0 \\
-0.0\end{array}\right)$ \\
\hline MBM 12-10 & $5.3\left(\begin{array}{c}+0.1 \\
-0.1\end{array}\right)$ & $1.9\left(\begin{array}{c}+2.1 \\
-0.9\end{array}\right)$ & $4.6\left(\begin{array}{l}+4.6 \\
-1.7\end{array}\right)$ & $0.8\left({ }_{-0.5}^{+2.2}\right)$ \\
\hline MBM 12-12 & $6.0\left(\begin{array}{l}+0.0 \\
-0.1\end{array}\right)$ & $5.5\left(\left(_{-0.1}^{+0.1}\right)\right.$ & $32.8\left(\begin{array}{l}+2.9 \\
-2.8\end{array}\right)$ & $0.1\left(\begin{array}{l}+0.1 \\
-0.0\end{array}\right)$ \\
\hline \multicolumn{5}{|c|}{$17-37 \mu \mathrm{m}$ region } \\
\hline MBM 12-2 & $0.1\left(\begin{array}{c}+0.1 \\
-0.0\end{array}\right)$ & $0.8\left(\begin{array}{c}+0.0 \\
-0.0\end{array}\right)$ & $1.8\left(\begin{array}{l}+0.1 \\
-0.1\end{array}\right)$ & $1.1\left(\begin{array}{c}+0.1 \\
-0.1\end{array}\right)$ \\
\hline MBM 12-3 & $1.2\left(\begin{array}{c}+0.1 \\
-0.1\end{array}\right)$ & $0.4\left(\left(_{-0.2}^{+0.3}\right)\right.$ & $4.8\left({ }_{-0.4}^{+0.4}\right)$ & $1.2\left(\begin{array}{c}+0.1 \\
-0.1\end{array}\right)$ \\
\hline MBM 12-4 & $0.2\left(\left(_{-0.1}^{+0.2}\right)\right.$ & $1.6\left(\left(_{-0.6}^{+0.8}\right)\right.$ & $2.8\left(\begin{array}{l}+0.8 \\
-0.5\end{array}\right)$ & $1.0\left(\begin{array}{c}+0.3 \\
-0.2\end{array}\right)$ \\
\hline MBM 12-5 & $0.7\left(\begin{array}{l}+0.2 \\
-0.1\end{array}\right)$ & $4.0\left(\begin{array}{l}+0.5 \\
-0.8\end{array}\right)$ & $3.5\left(\begin{array}{l}+1.0 \\
-0.9\end{array}\right)$ & $0.9\left(\begin{array}{c}+0.7 \\
-0.3\end{array}\right)$ \\
\hline MBM 12-6 & $1.5\left(\begin{array}{l}+0.4 \\
-0.2\end{array}\right)$ & $0.1\left(\begin{array}{l}+0.0 \\
-0.0\end{array}\right)$ & $7.1\left(\begin{array}{l}+1.5 \\
-1.3\end{array}\right)$ & - \\
\hline MBM 12-10 & $0.4\left(\begin{array}{l}+0.9 \\
-0.3\end{array}\right)$ & - & $2.9\left({ }_{-1.5}^{+3.1}\right)$ & - \\
\hline MBM 12-12 & $0.6\left(\left(_{-0.2}^{+0.3}\right)\right.$ & $0.8\left({ }_{-0.2}^{+0.2}\right)$ & $3.3\left(\begin{array}{l}+1.1 \\
-0.7\end{array}\right)$ & $0.9\left(\begin{array}{c}+0.2 \\
-0.1\end{array}\right)$ \\
\hline
\end{tabular}

${ }^{a}$ Mass-averaged size of the amorphous and crystalline silicates.

${ }^{b}$ Mass fraction of the crystalline grains (ratio of crystalline silicate mass to total silicate mass).

${ }^{c}$ Mass ratio of forsterite to enstatite.

for our sample of T Tauri stars, and also found evidence for grain growth in the protoplanetary discs of the MBM 12 members.

Kessler-Silacci et al. (2006, 2007) and Apai et al. (2005) noticed that later type TTS have weaker 10 micron features than earlier type TTS, and related this to the location of the dust causing this feature. This relation was confirmed by Sicilia-Aguilar et al. (2007), who found that the presence of a very weak feature was 3 times more frequent for M-type stars than for earliertype stars. The temperature of the silicates causing the feature is $\sim 300 \mathrm{~K}$, a temperature that is reached more inwards for lower luminosity sources than for higher luminosity sources: under the assumption of a similar disc structure for all spectral types discussed here, an M0-type star reaches $300 \mathrm{~K}$ between 0.7 and 1.5 AU, while it is between 1.2 and $3 \mathrm{AU}$ for a K5-type star, a factor 2 difference in distance. Furthermore, the density distribution has a radial dependence, as it decreases with increasing radius, and grain growth occurs faster in more dense environments. This implies that grain growth will be faster in the more inward regions, so that lower luminosity sources will appear to have larger grains, as their 10 micron feature originates from a denser region, in which growth naturally occurs more rapidly. This is also seen in our spectra: in Fig. 6, we plot the continuumnormalised spectra, and also list their spectral types. With the exception of MBM 12-4, the fainter objects indeed have weaker features. This is further quantified through our fitting with the TLTD method: in Fig. 7, we show the relation between the massaveraged grains size in the 10 micron region and the effective temperature of the central star. There is a clear trend for cooler stars to have larger grain sizes.

Of course, not all discs need to have the same structure, and the degree of flaring can also play an important role in this context. However, to study these effects, detailed radiative transfer modelling of each source is needed, which is beyond the scope of this paper.

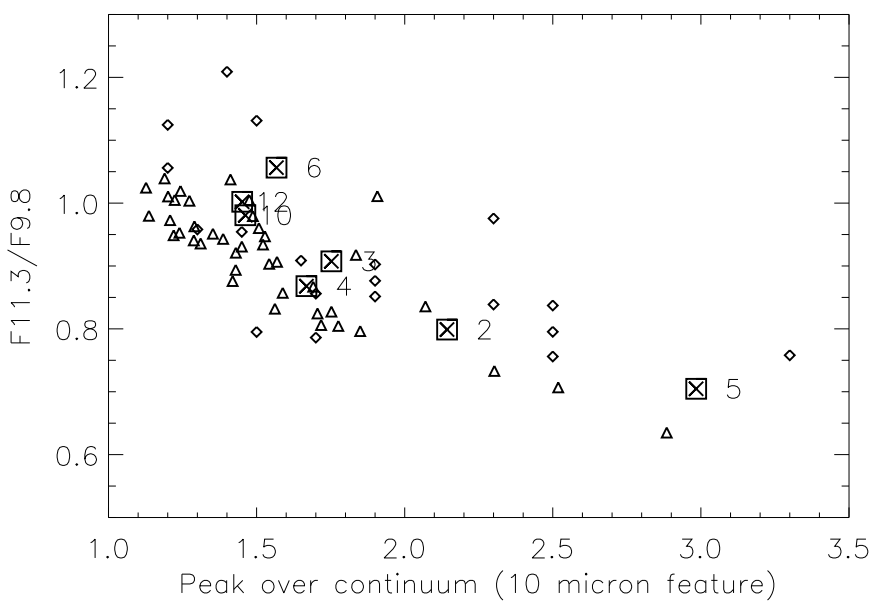

Fig. 5. Relation between the shape of the $10 \mu \mathrm{m}$ feature (flux ratio 11.3 over $9.8 \mu \mathrm{m}$ ) and the feature strength (peak over continuum). Our objects (squared symbols, numbered as listed in Table 1), follow the trend observed for TTS in Tr 37 (4 Myr) and NGC 7160 (12 Myr), shown by diamonds (Sicilia-Aguilar et al. 2007) and TTS in Taurus (2 Myr), shown by triangles (Pascucci et al. 2008): the flux ratio is correlated with the feature strength.

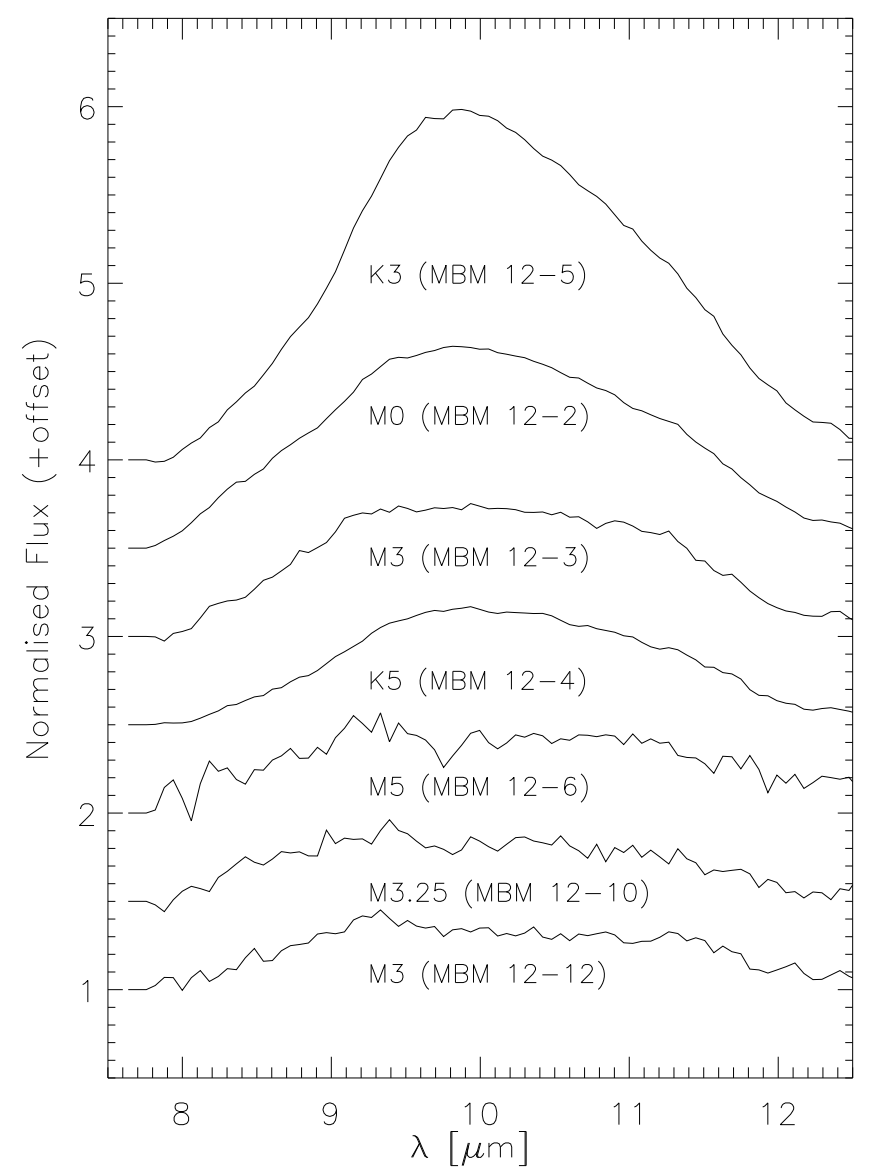

Fig. 6. Continuum normalised flux in the $10 \mu \mathrm{m}$ region. The objects with the latest spectral types tend to have the weakest feature.

\subsection{Crystallisation}

The dust that is initially incorporated into the protoplanetary disc is largely amorphous, as it comes from the ISM, for which an upper limit of $\sim 2 \%$ in mass of the crystalline grains was determined using spherical grains (Kemper et al. 2004). 


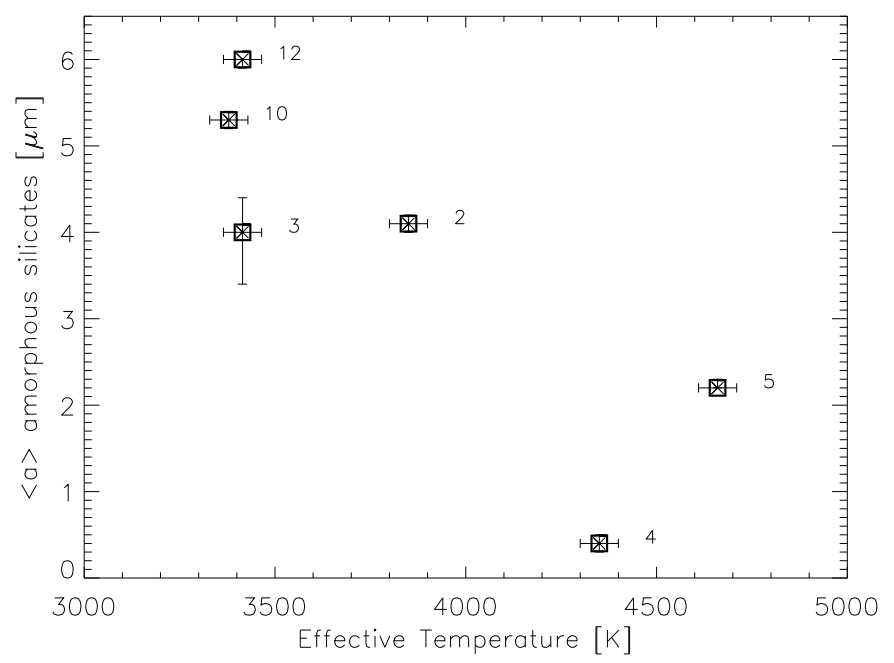

Fig. 7. Mass-averaged size of the amorphous silicate grains in the warmer region, versus effective temperature of the central star. The cooler the star (the later the spectral type), the larger the dust grains tend to be.

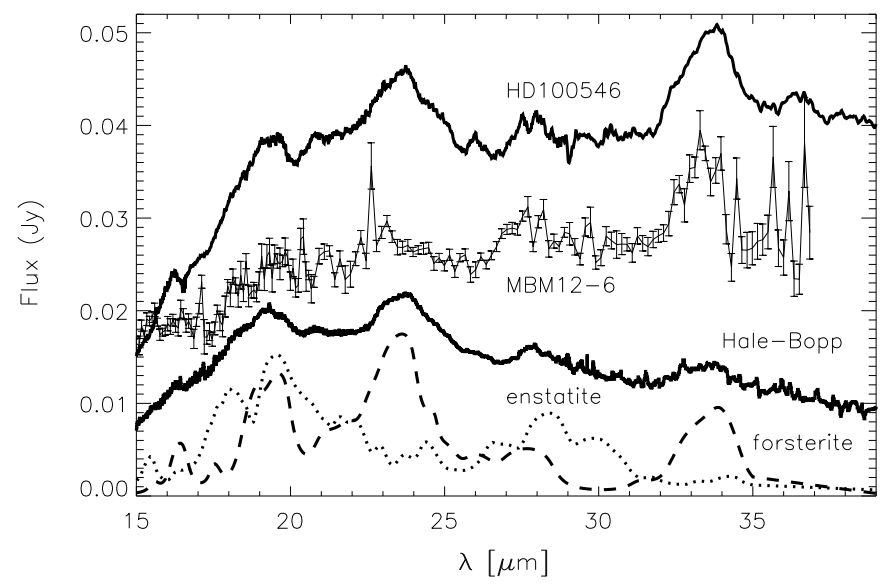

Fig. 8. MBM 12-6, host of a large amount of crystalline silicates, witnessed by the agreement in peak positions between the spectrum and the features of the mass absorption coefficients of enstatite (Jäger et al. 1998; dotted line) and forsterite (Servoin et al. 1973; dashed line). For comparison, we also show the Herbig Be star HD100546 (Malfait et al. 1998), and the solar-system comet Hale-Bopp (Crovisier et al. 1997), both hosts of large amounts of crystalline dust. The fluxes of HD 100546 and Hale-Bopp are scaled to match the flux of MBM 12-6.

Min et al. (2007) further improved this number using grains with irregular shapes and derived a crystallinity of only $\sim 1 \%$. However, the amorphous dust may become crystalline through thermal annealing (e.g. Fabian et al. 2000) or shock heating (e.g. Scott \& Krot 2005). We derived the crystalline mass fraction in the shorter wavelength region, and found a large degree of variation: between $2.9_{-1.1}^{+1.2} \%$ for MBM 12-5 and $47_{-17}^{+25} \%$ for MBM 126 . The crystalline fraction of MBM 12-6 is not very well determined, therefore we use the lower limit of $30 \%$ that was found for this object. The mass fraction of the crystalline silicates is not related to the spectral type. Furthermore, we do not find a correlation between crystallinity and the size of the amorphous grains; also the size of the crystalline grains appears to be uncorrelated with amorphous grain size (see Fig. 9). However, we do note that the sources with the largest crystalline mass fractions (MBM 12-6 and MBM 12-12) also have the largest grains size, both for the amorphous as for the crystalline grains (see

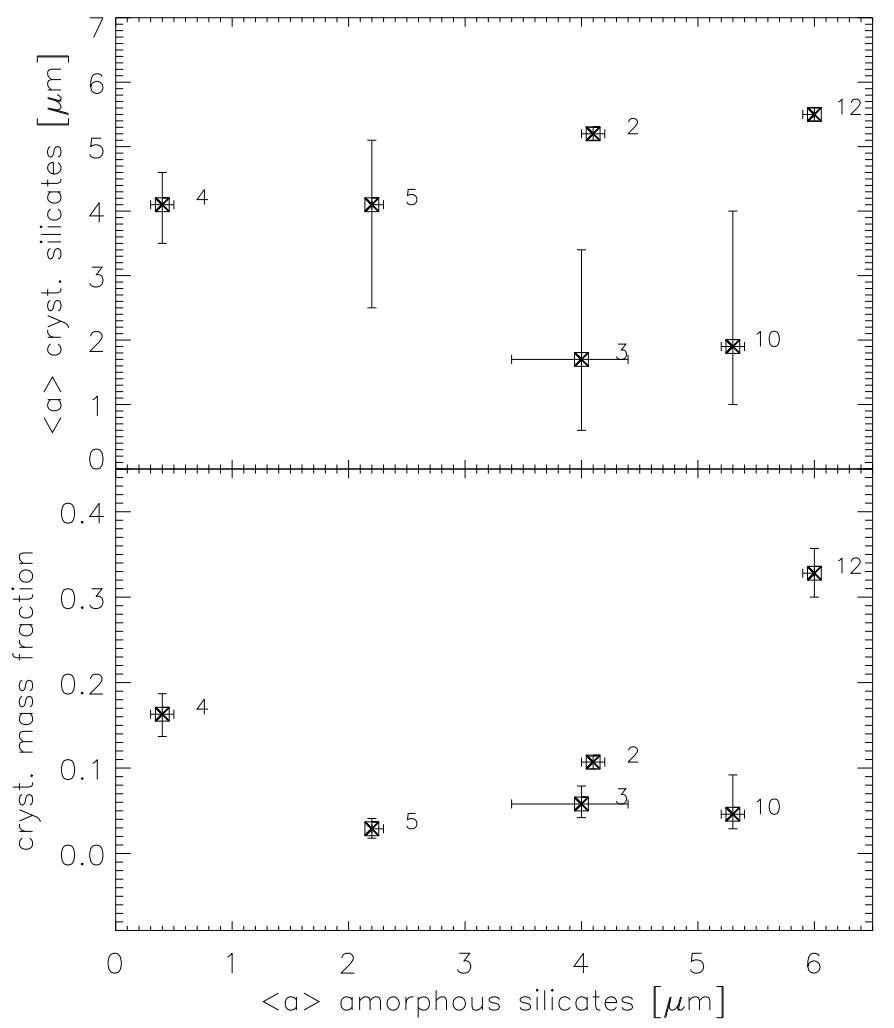

Fig. 9. Relation between the properties of the crystalline silicates and the size of the amorphous silicates in the 7 to 17 micron region. Upper panel: mass-averaged size of the crystalline silicates versus massaveraged size of the amorphous silicates. The two appear unrelated. Lower panel: crystalline mass fraction versus mass-averaged size of the amorphous silicates. Also here we do not see a correlation.

also Fig. 10). In the longer wavelength region, we find the variation in crystallinity to be less, and the crystalline mass fraction smaller: between $1.8_{-0.1}^{+0.1} \%$ for MBM $12-2$ and $7.1_{-1.3}^{+1.5} \%$ for MBM 12-6. This difference in crystallinity between both regions for the whole sample could be related to the presence of a large amount of small amorphous silicate grains in the cooler disc region (average size for the sample is $0.7 \mu \mathrm{m}$ ), while in the warmer disc region the amorphous silicate grains are much larger (average size for the sample is $3.7 \mu \mathrm{m}$ ). Larger grains will produce less detectable features, so that the crystalline grains that still show features will appear more abundant.

In Fig. 8, we show the 15 to $37 \mu \mathrm{m}$ spectrum of MBM 12-6, the object with the largest fraction of crystalline grains. Its IR spectral appearance is similar to that of HD 100546, a Herbig B9e star with highly evolved dust, which is remarkable, given that their effective temperatures are very different: $11000 \mathrm{~K}$ for the B9 star, and only $3200 \mathrm{~K}$ for the M5 type star. Still, the dust around both objects is dominated by crystalline grains, indicating that the temperature of the central star does not play an important role in the crystallisation process. The spectrum of MBM 12-6 is also similar to that of solar-system comet HaleBopp, so that similar dust processing mechanisms must exist in both our solar system and the disc of MBM 12-6.

In the dust model by Gail (1998), the composition of the dust is derived based on condensation sequences and chemical equilibrium considerations. He predicts that - assuming crystalline silicates form as high temperature gas phase condensates - of the crystalline silicates, enstatite will be the dominant constituent, while forsterite is only present in a small region close to the star. 


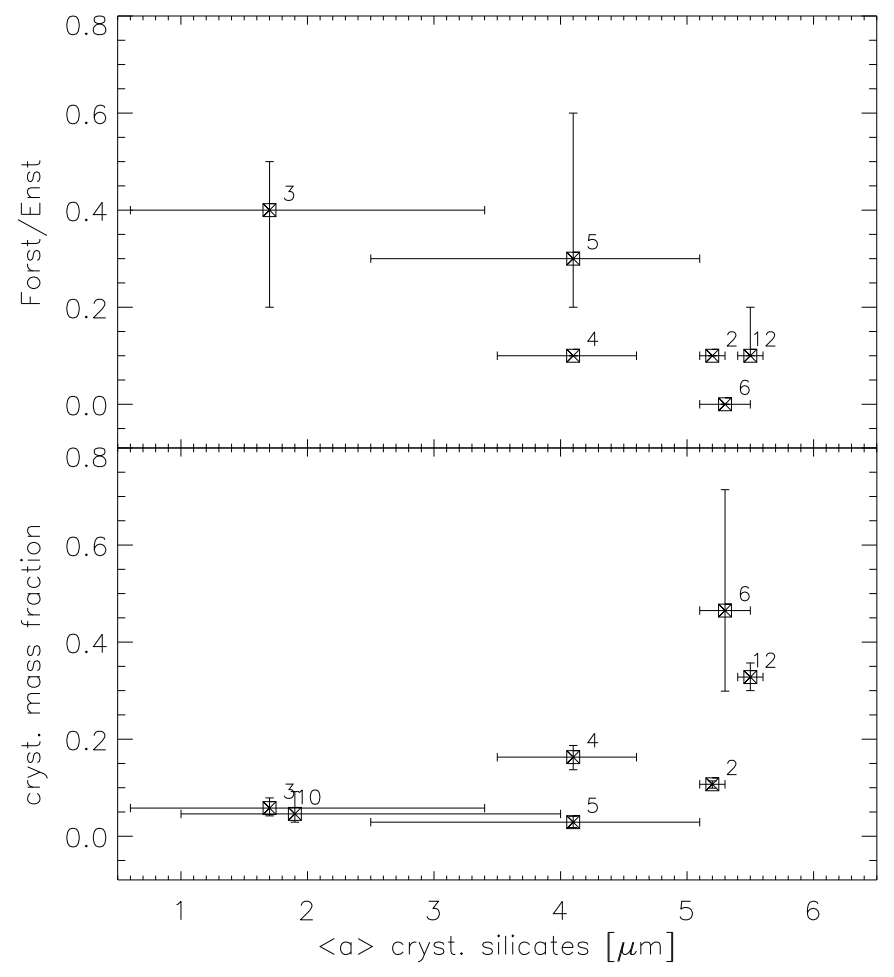

Fig. 10. Properties of the the crystalline silicates, derived from the 7 to 17 micron region. Upper panel: the forsterite over enstatite ratio as a function of the size of the crystalline silicates. There is a trend for larger grains to have a smaller forst/enst ratio. Lower panel: crystallinity in function of crystalline grain size. The crystalline fraction only exceeds $20 \%$ when the crystalline grains are larger than 5 micron.

More recent simulations show that radial mixing within the disc is capable of transporting a substantial amount of crystalline material from the inner disc towards more colder, outward regions (Keller \& Gail 2004).

To better understand the crystallisation process occurring in the $\mathrm{T}$ Tauri discs, we look at the forsterite to enstatite ratio. In Table 5, we list the forsterite to enstatite mass ratio for both regions. In the warmer region, the smallest ratio is found for those sources with the largest grains (see Fig. 10), implying that enstatite is more abundant (relative to forsterite) when larger grains are present. We further compare the relative mass fractions of forsterite and enstatite in both regions: the average ratio for the sample is 0.3 in the warmer region, and 0.9 in the cooler region.

It is thus clear that there is a spatial gradient in the forsterite to enstatite ratio, with forsterite dominating the cooler regions, and enstatite more abundant in the warmer, inner regions. Such a gradient was already found in a few earlier studies, e.g. Bouwman et al. (2008). This is in contrast with the predictions by Gail $(1998,2004)$, suggesting that the chemical equilibrium conditions needed for the forsterite to enstatite conversion are not reached inside these discs, and that the crystallisation process must be different, even when including radial mixing. It is interesting to consider that enstatite is more abundant in the warmer (inner) regions, and that we also find a higher enstatite abundance in those sources that have larger grains (see Fig. 10). These observations suggest that enstatite forms more easily in regions of higher density, where also grain growth is more abundant. Bouwman et al. (2008) discuss the formation of enstatite and forsterite in detail, and link the formation of enstatite in the inner region with the conditions that prevail there: due to the higher density and temperature, it takes the dust grains longer
Table 6. The logarithm of the IR excess over the photosphere.

\begin{tabular}{lcccc}
\hline \hline Object & $\log$ & $\log$ & $\log$ & $\log$ \\
$\left(F_{10} / F_{*, 10}\right)$ & $\left(F_{24} / F_{*, 24}\right)$ & $\left(F_{30} / F_{*, 30}\right)$ & $\left(F_{70} / F_{*, 70}\right)$ \\
\hline MBM 12-1 & 0.0 & 0.0 & 0.0 & $<2.1$ \\
MBM 12-2 & 1.5 & 2.0 & 2.0 & $<3.2^{a}$ \\
MBM 12-3 & 0.8 & 1.3 & 1.4 & $<3.0^{a}$ \\
MBM 12-4 & 1.5 & 2.2 & 2.1 & 3.2 \\
MBM 12-5 & 1.0 & 1.6 & 1.6 & 2.6 \\
MBM 12-6 & 0.9 & 1.6 & 1.7 & $<2.8$ \\
MBM 12-7 & $<0$ & $<0.6$ & $<1.2$ & $<2.9$ \\
MBM 12-8 & $<0.4$ & $<0.8$ & $<1.5$ & $<3.3$ \\
MBM 12-9 & $<0.2$ & $<0.8$ & $<1.7$ & $<3.2$ \\
MBM 12-10 & 0.9 & 1.4 & 1.4 & $<2.7$ \\
MBM 12-11 & $<0.1$ & $<0.6$ & $<1.6$ & $<3.0$ \\
MBM 12-12 & 0.6 & 1.2 & 1.3 & $<2.1$ \\
\hline
\end{tabular}

${ }^{a}$ Combined flux of $\mathrm{LkH} \alpha 262$ and 263.

to cool down so that, potentially, equilibrium conditions can be reached - which is more unlikely at larger radial distances, where both the density and the temperature are lower.

\subsection{Disc properties}

\subsubsection{Disc fraction}

We obtained Spitzer data for the complete sample of MBM 12 members; 4 of them, however, could not be detected. The remaining 8 objects (spectral type range K3 to M5) have a disc fraction rate of 7 out of 8 , or nearly $90 \%$. This is very high, when compared to a similar spectral type range in other star forming regions: Damjanov et al. (2007) derive a disc fraction of $47 \%$ for M0-M4 and 55\% for K3-K8 type stars in the 2 Myr-old Chamaeleon I and 63\% region; however, Flaherty \& Muzzerole (2008) derive a disc fraction between 65 and $81 \%$ for the $2 \mathrm{Myr}$ old clusters NGC 2068 and NGC 2071. The high disc fraction rate found for MBM 12 may (partly) be attributed to the absence of close companions to the disc bearing stars: there are no companions found at a projected distance smaller than 0.'39 (or $70 \mathrm{AU}$ at the distance of MBM 12).

Of the $7 \mathrm{~T}$ Tauri stars with a disc, 3 are candidate transitional objects, which is a high rate for a region with such a young age ( $2 \mathrm{Myr}$ ), pointing to fast inner disc dispersal. However, due to the lack of data between 3.5 and $8 \mu \mathrm{m}$, we cannot further discuss the differences in inner disc structure; additional observations could shed more light on this topic. For the four non-detected members, we derived upper limits, to put some constraints on their disc properties. In all 4 cases, the upper limit at 10 micron does not allow for an IR excess to be present, suggesting that, if these objects do have a disc - which cannot be excluded from the upper limits derived at longer wavelengths - these discs must have inner holes. In Table 6, we list the excess luminosity over the stellar photosphere; the data are visualised in Fig. 11, where we plot the excesses versus the effective temperature. Those sources that have the largest excesses also have the highest temperatures, although there is no linear relation between both quantities.

\subsubsection{The influence of companions}

The radiation of cold, outer discs was detected by Hogerheijde et al. (2003) at 450 and $850 \mu \mathrm{m}$ for 4 stars in MBM 12: $\mathrm{LkH} \alpha 262$ (MBM 12-2), $\mathrm{LkH} \alpha 263$ ABC (MBM 12-3), $\mathrm{LkH} \alpha 264 \mathrm{~A}$ (MBM 12-4) and S18 ABab (MBM 12-12). These authors derived disc masses between 0.005 and $0.23 \times 10^{-2} M_{\odot}$ 


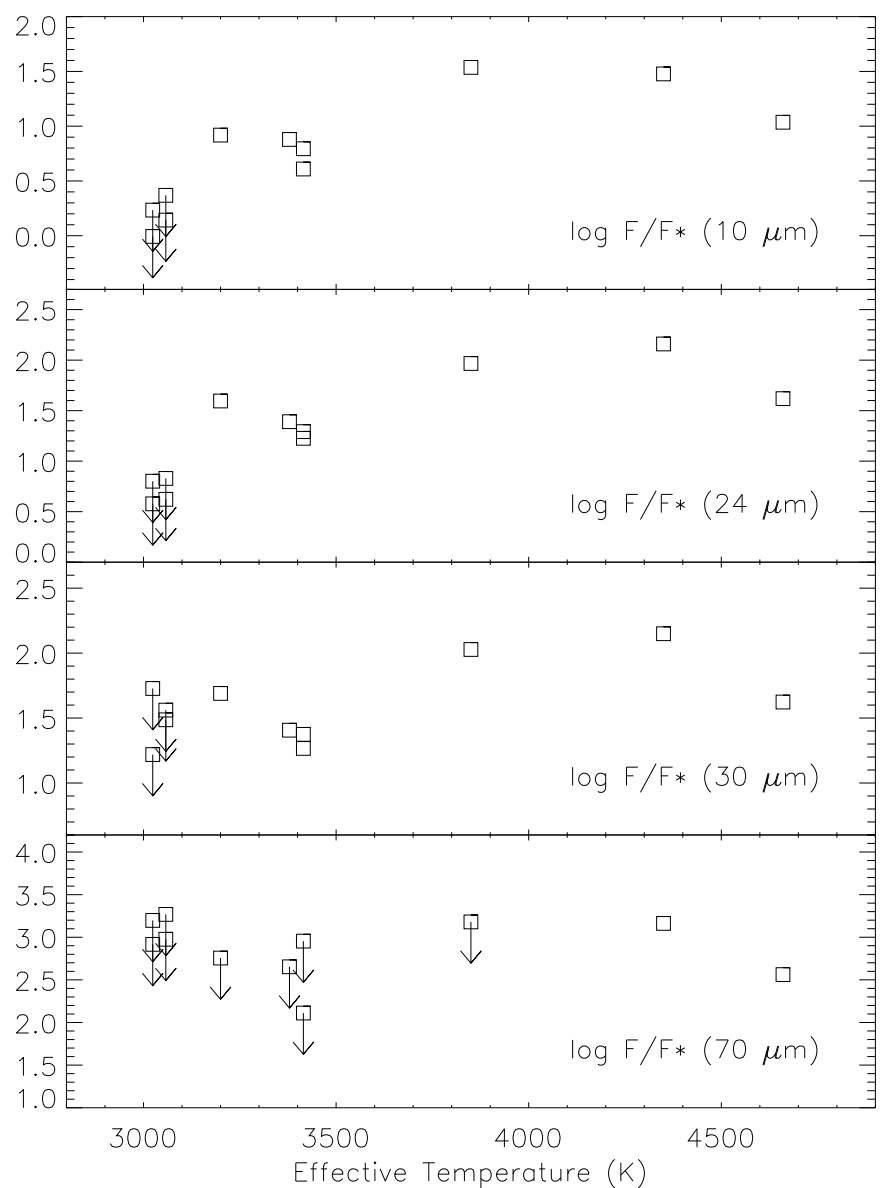

Fig. 11. The logarithm of the IR excess at selected wavelengths, plotted as a function of the effective temperature of the central star. Upper limits are indicated with arrows. The warmer the star, the larger the infrared excess - apart for the warmest object, MBM 12-5, with a temperature of $4660 \mathrm{~K}$.

for these 4 sources, and noticed a relation between the cold disc masses (as derived from the submm photometry) and the distance of the companion: the 2 stars with separations of 100-200 AU have much lower disc masses than the 2 stars with separations of 2000-4000 AU. Artymowicz \& Lubow (1994) discussed disc truncation by companions, and showed that the tidal limit for a disc around a member of a binary is $\sim 0.4$ times the separation. For objects with a not too wide companion, relative to their disc size, this means that the companion will effectively truncate their disc. Jensen et al. (1996) showed with millimetre observations that indeed objects with separations less than 100 AU have lower disc masses, when compared to their single counterparts, or to those objects which have only wider companions.

We now want to see what the influence of companions is on the "middle" region of the disc that can be observed in the mid-IR. Therefore, we calculated the IR excess at 30 micron for 2 groups we defined in our sample: 1) the "binaries", including binaries with projected separations smaller than $400 \mathrm{AU}$ (MBM 12-3, 5, 10 and 12); and 2) the "singles", including single stars and binaries with larger projected separations (MBM 12-2, 4 and 6). This gives average excess values of $F_{30} / F_{* 30} \sim 25$ for the binaries, and $F_{30} / F_{*, 30} \sim 80$ for the singles, indicating that the discs around the binaries are indeed influenced by their

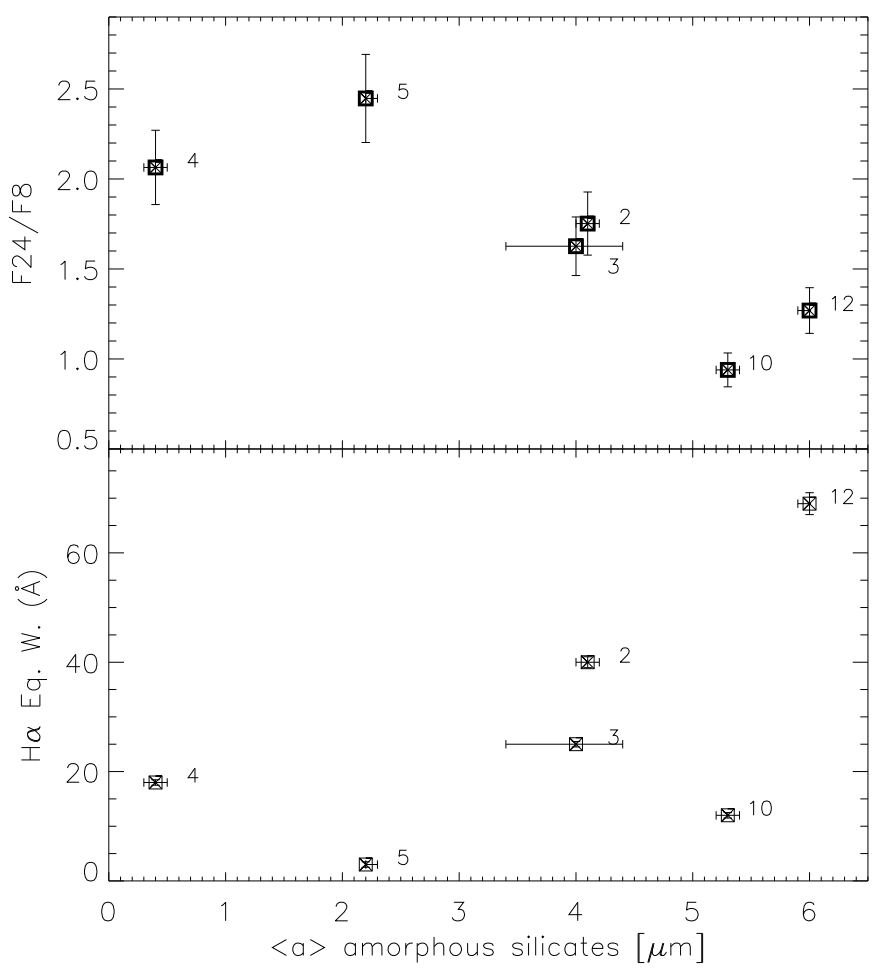

Fig. 12. Disc properties related to the amorphous grain size derived from the warmer region. Top panel: degree of flaring (derived from the flux ratio at 24 and $8 \mu \mathrm{m}$ ) versus the mass-averaged grain size. The larger the grains, the smaller the flaring, which suggests dust settling when grains have grown. Lower panel: relation between the equivalent width of $\mathrm{H} \alpha$ and the mass-averaged size of the amorphous grains. The trend suggests that the higher the accretion rate, the larger the grains in the disc atmosphere are.

companions, as their excess is a factor of 3 less than that of the single stars.

Bouwman et al. (2006) studied a sample of T Tauri stars in the 8 Myr-old cluster $\eta \mathrm{Cha}$, and found that only 1 out of 6 known or suspected binaries (all with projected separations $\leq 20 \mathrm{AU}$ ) retain a protoplanetary disc. Conversely, 7 out of 9 stars believed to be single have discs, suggesting that binary discs have shorter lifetimes. Our MBM 12 sample does not include such close companions around the disc-bearing stars, but nevertheless shows a dependence of its dust characteristics on the presence of a companion. It is interesting to see that the two groups (binaries and singles) already differ in disc properties at such a young age, even when there are no very close companions involved.

\subsubsection{Disc flaring}

The classical model of a T Tauri disc is one in which the optically thick midplane (containing the bulk of the disc mass) is surrounded by an optically thin disc atmosphere, which is hot and is the source of the infrared emission features. The disc can flare under hydrostatic equilibrium, and when the gas and dust are well-mixed, the turbulent motion of the gas will support the dust grains in the disc atmosphere against gravitational settling towards the disc midplane. In the disc evolution model from Dullemond \& Dominik (2004), grains located in the atmosphere of a flared disc that grow to a certain size can no longer be supported by the gas. As a consequence, the larger grains will settle towards the disc midplane, hereby reducing the amount of 
flaring in the disc. In Fig. 12, we plot the ratio of the fluxes at 24 and $8 \mu \mathrm{m}$ versus the derived grain size of the amorphous silicates. This flux ratio can be used as a proxy for the degree of flaring in a disc (e.g. Apai et al. 2005; Scholz et al. 2007, for brown dwarfs and Furlan et al. 2005 for TTS): the larger the ratio, the more flared the disc. It is clear that the more flared the MBM 12 discs are, the smaller the derived silicate grain sizes, in agreement with the dust settling model from Dullemond \& Dominik (2004).

\subsubsection{Accretion rate}

We only have two weak-line T Tauri stars in our detected sample: MBM 12-1, which has no excess emission, and MBM 12-10, which has an IR excess due to warm dust. We thus see accretion in all but one of the objects which have an IR excess. SiciliaAguilar et al. (2007) showed a particular relation between the accretion rate derived from the $U$-band emission, and the grain sized derived from the $10 \mu \mathrm{m}$ feature: they found that objects with higher accretion rates have, in general, larger dust grains. This suggests that turbulence supports large dust grains against settling, and that when the accretion weakens, it will become apparent in the observed grain size. In Fig. 12, we plot the equivalent width of the $\mathrm{H} \alpha$ line as a function of the derived grain size, and we find a similar tendency for our T Tauri stars - apart from object MBM 12-10, which is a WTTS. It is thus tempting to assume that the larger turbulence, the easier it is to support large grains against settling towards the disc midplane. Caution should be taken, however, given 1) the variability of the $\mathrm{H} \alpha$ line; and 2) the dependency of the equivalent width for a given accretion rate on spectral type. Furthermore, some weak-line TTS - based on the $\mathrm{H} \alpha$ equivalenth width - were found to be accreting, through the analysis of the width of their broad $\mathrm{H} \alpha$ line (Sicilia-Aguilar et al. 2006). Therefore, additional studies, where the line is observed at several epochs and with higher spectral resolution (so that the accretion rate can be derived from the $10 \%$ velocity width instead of the equivalent width), are required to better understand this relation.

\section{Conclusions}

We presented Spitzer spectroscopy and photometry for the complete sample of $\mathrm{T}$ Tauri stars in the 2 Myr-old star forming cloud MBM 12. From the 12 objects observed, 8 were detected and could be studied more in detail in this paper. We composed spectral energy distributions for our sample, and found that only 1 object shows no excess at all, and is probably a discless T Tauri star. The other objects show excesses that can be attributed to the presence of a protoplanetary disc. Of the 7 IR excess sources, 3 have candidate transitional discs.

We analysed the properties of the warm dust (visible through their solid-state bands in the infrared) with the two-layer temperature distribution method from Juhász et al. (2009) and derived the mass fraction of the different species, and their massaveraged sizes. We showed that the dust components seen in other young stars can very well explain the spectral features observed in MBM 12 discs. We also found evidence for grain growth: the shape and the strength of the $10 \mu \mathrm{m}$ feature shows that grains with submicron sizes, as well as grains of sizes up to a few microns are present in the discs in varying amounts. The presence of crystalline silicates demonstrates that hightemperature processes take place early around other stars, consistent with meteorite evidence from the young solar system. In a next step, we related the derived properties with the stellar and disc properties, and found:

1. The later the spectral type of the central object, the weaker the $10 \mu \mathrm{m}$ feature, implying larger dust grains. This is most likely a luminosity effect, as in less luminous stars, the radial distance observed at $10 \mu \mathrm{m}$ is smaller, hence the density higher and, as a consequence, faster grain growth is seen - this does not need to imply a real difference in the size distribution.

2. A spatial gradient in grain growth: the mass-averaged grain sizes are larger in the warmer region than in the cooler region, which also can be related to a difference in density.

3. There is a large spread in the degree of crystallisation (between 3 and $>30 \%$ ), despite a similar age for the objects; the crystallisation fraction is independent of spectral type.

4. The crystallisation is also independent of grain growth: neither the mass fraction in crystalline grains nor the size of the crystalline grains are correlated with the size of the amorphous silicates. However, the largest crystalline fractions are observed for those sources with largest grain sizes.

5. A spatial gradient in crystalline composition, as observed in the forsterite to enstatite mass ratio: forsterite is much more dominant in the outer than in the inner disc region. Enstatite is more abundant in those sources with larger grains, and in regions where the density is higher. This suggests that its formation process needs higher densities than forsterite.

6. The presence of a spatial gradient in both grain sizes and composition indicates that radial mixing is not a very efficient process in these protoplanetary discs.

7. Companions, even at distances greater than $70 \mathrm{AU}$, influence disc evolution: the IR excess of single sources is a factor of 3 higher than that of binaries.

8. Disc flaring and grain size are mildly related: the more the discs flare, the smaller the grains in their atmosphere.

9. There is a tendency for objects with a stronger $\mathrm{H} \alpha$ line to have larger grains, suggesting that more heavily accreting (more turbulent) sources can support larger grains in their disc atmosphere more efficiently.

We are aware that these findings are based on a small sample, and that it should be compared with a larger database of wellknown T Tauri stars for which infrared spectra of high quality are available. This study is part of a larger programme with Spitzer on discs in young clusters. The low-mass M-type stars in the Coronet cluster have already been discussed in Sicilia-Aguilar et al. (2008), while T Tauri stars in the $\eta$ Cha cluster will be presented by Sicilia-Aguilar et al. (in preparation) and T Tauri stars in the $\epsilon$ Cha cluster by Fang et al. (in preparation). From a comparison of the results in the different clusters, we will be able to better determine the relations between the different stellar and dust properties, so that they can be incorporated in theoretical (evolutionary) disc models of young objects.

Acknowledgements. It is a pleasure to thank Ray Jayawardhana, who sparked G.M.'s interest in this cloud, Leen Decin for calculating the appropriate MARCS models for our sample and Jeff Nichols for help with Python. We also thank the referee, Dan Watson, for his prompt report. G.M. and A.S.-A. acknowledge support by the Deutsche Forschungsgemeinschaft, DFG, project numbers ME 2061/3-2 and SI 1486/1-1, respectively. W.L. asknowledges support from the Australian Academy of Sciences international exchange program. We made extensively use of the SAO/NASA Astrophysics Data System and SIMBAD hosted by CDS, Strasbourg. 


\section{Appendix}

Table 7. Parameters involved in the TLTD model.

\begin{tabular}{ll}
\hline \hline Parameter & Meaning \\
\hline$F_{v}$ & Observed flux \\
$F_{v, \text { cont }}$ & Total continuum flux \\
$d$ & Distance to the source \\
$R_{*}$ & Stellar radius \\
$T_{*}$ & Stellar temperature \\
$D_{0}$ & Contribution of the star to the total flux \\
$D_{1}$ & Contribution of the inner rim to the total flux \\
$D_{2}$ & Contribution of the midplane to the total flux \\
$D_{i, j}$ & Mass contribution of species $i$ with size $j$ \\
$T_{a, \text { out }}$ & Lowest temperature in the disk atmosphere \\
$T_{a, \text { in }}$ & Highest temperature in the disk atmosphere \\
$T_{r, \text { out }}$ & Lowest temperature in the inner rim \\
$T_{r, \text { in }}$ & Highest temperature in the inner rim \\
$T_{m, \text { out }}$ & Lowest temperature in the disk midplane \\
$T_{m, \text { in }}$ & Highest temperature in the disk midplane \\
$\kappa_{i, j}$ & Mass absorption coefficient of species $i$ with size $j$ \\
$q r$ & Power exponent of the temperature distribution (as a function of radius) in the rim \\
$q a$ & Power exponent of the temperature distribution (as a function of radius) in the disk atmosphere \\
$q m$ & Power exponent of the temperature distribution (as a function of radius) in the disk midplane \\
\hline
\end{tabular}

Table 8. For each object, we list the reduced $\chi^{2}$ of the fit, and the mass fraction of each species for the 3 different sizes, used to fit the shorter and longer wavelengths regions (upper and lower part of the table, respectively). We only show mass fractions that are higher than $5 \%$.

\begin{tabular}{|c|c|c|c|c|c|c|c|c|}
\hline & & "MBM 12-2 & "MBM 12-3 & "MBM 12-4 & MBM 12-5 & $\overline{\mathrm{MBM}} 12-6$ & $\overline{\mathrm{MBM}} 12-10$ & MBM 12-12 \\
\hline 7-17 micron range & $\chi^{2}$ & 12.2 & 5.7 & 9.0 & 9.7 & 4.9 & 3.4 & 5.6 \\
\hline Species & Size & \multicolumn{7}{|c|}{ Mass fractions (in percentage) } \\
\hline Amorphous silicate & $0.1 \mu \mathrm{m}$ & $13.2\left(\begin{array}{c}+0.4 \\
-0.4\end{array}\right)$ & - & $48.2\left(\begin{array}{l}+2.3 \\
-2.1\end{array}\right)$ & $43.4\left(\begin{array}{l}+0.4 \\
-0.4\end{array}\right)$ & - & - & - \\
\hline (Olivine type) & $1.5 \mu \mathrm{m}$ & -0.4 & - & - & -0.4 & - & - & - \\
\hline & $6.0 \mu \mathrm{m}$ & - & $29.7\left(\begin{array}{l}+13.1 \\
-14.2\end{array}\right)$ & - & - & $8.8\left({ }_{-8.7}^{+27.6}\right)$ & $17.6\left(\begin{array}{c}+29.2 \\
-15.7\end{array}\right)$ & $53.3\left(\begin{array}{l}+7.5 \\
-9.5\end{array}\right)$ \\
\hline Amorphous silicate & $0.1 \mu \mathrm{m}$ & - & - & $15.9\left(\begin{array}{c}+3.9 \\
-2.8\end{array}\right)$ & - & - & - & - \\
\hline (Pyroxene type) & $1.5 \mu \mathrm{m}$ & $20.2\left(\begin{array}{c}+0.8 \\
-0.8\end{array}\right)$ & $35.2\left(\begin{array}{c}+9.7 \\
-7.9\end{array}\right)$ & $16.8\left(\begin{array}{c}+3.2 \\
-3.3\end{array}\right)$ & $24.0\left(\begin{array}{c}+0.6 \\
-0.9\end{array}\right)$ & - & $13.8\left(\begin{array}{l}+3.3 \\
-3.3\end{array}\right)$ & - \\
\hline \multirow{4}{*}{ Forsterite } & $6.0 \mu \mathrm{m}$ & $55.7\left(\begin{array}{c}+0.9 \\
-1.0\end{array}\right)$ & $19.8\left(\begin{array}{c}+18.6 \\
-12.9\end{array}\right)$ & - & $29.0\left({ }_{-1.2}^{+1.4}\right)$ & $32.0\left(\begin{array}{c}+34.3 \\
-30.5\end{array}\right)$ & $62.0\left(\begin{array}{c}+16.0 \\
-28.4\end{array}\right)$ & $10.9\left(\begin{array}{l}+9.7 \\
-8.3\end{array}\right)$ \\
\hline & $0.1 \mu \mathrm{m}$ & - & - & - & - & - & - & - \\
\hline & $1.5 \mu \mathrm{m}$ & - & - & - & - & - & - & - \\
\hline & $6.0 \mu \mathrm{m}$ & - & - & - & - & - & - & - \\
\hline \multirow[t]{3}{*}{ Enstatite } & $0.1 \mu \mathrm{m}$ & - & - & - & - & - & - & - \\
\hline & $1.5 \mu \mathrm{m}$ & - & - & - & - & - & - & - \\
\hline & $6.0 \mu \mathrm{m}$ & $9.0\left(\begin{array}{c}+0.8 \\
-0.9\end{array}\right)$ & - & $10.6\left(\begin{array}{l}+3.1 \\
-3.2\end{array}\right)$ & - & $40.1\left(\begin{array}{l}+19.7 \\
-13.1\end{array}\right)$ & - & $29.1\left(\begin{array}{l}+2.6 \\
-26\end{array}\right)$ \\
\hline \multirow[t]{3}{*}{ Silica } & $0.1 \mu \mathrm{m}$ & -0.9 & - & - & - & - & - & - \\
\hline & $1.5 \mu \mathrm{m}$ & - & - & - & - & - & - & - \\
\hline & $6.0 \mu \mathrm{m}$ & - & $7.0\left({ }_{-2.2}^{+2.9}\right)$ & - & - & $5.4\left({ }_{-4.6}^{+6.6}\right)$ & - & - \\
\hline 17-37 micron range & $\chi^{2}$ & 12.3 & 8.3 & 14.0 & 14.2 & 5.9 & 3.6 & 5.7 \\
\hline Species & Size & \multicolumn{7}{|c|}{ Mass fractions (in percentage) } \\
\hline \multirow{3}{*}{$\begin{array}{l}\text { Amorphous silicate } \\
\text { (Olivine Type) }\end{array}$} & $0.1 \mu \mathrm{m}$ & $65.2\left(\begin{array}{c}+2.9 \\
-4.7\end{array}\right)$ & - & $51.1\left(\begin{array}{c}+5.6 \\
-9.6\end{array}\right)$ & - & - & $37.6\left(\begin{array}{l}+13.1 \\
-15.4\end{array}\right)$ & $30.5\left(\begin{array}{l}+17.0 \\
-2.5\end{array}\right)$ \\
\hline & $1.5 \mu \mathrm{m}$ & - & $68.8\left(\begin{array}{c}+3.8 \\
-4.6\end{array}\right)$ & $6.4\left(\begin{array}{c}+10.4 \\
-5.3\end{array}\right)$ & $36.7\left({ }_{-4.9}^{+2.6}\right)$ & - & - & $32.7\left(\begin{array}{c}+19.0 \\
-14.3\end{array}\right)$ \\
\hline & $6.0 \mu \mathrm{m}$ & - & - & - & - & - & - & - \\
\hline Amorphous silicate & $0.1 \mu \mathrm{m}$ & $30.4\left(\begin{array}{c}+3.6 \\
-4.2\end{array}\right)$ & $21.0\left(\begin{array}{c}+3.6 \\
-6.2\end{array}\right)$ & $33.0\left({ }_{-3.2}^{+1.6}\right)$ & $47.6\left({ }_{-13.6}^{+3.8}\right)$ & $8.1\left({ }_{-7.9}^{+58.2}\right)$ & $41.7\left({ }_{-14.4}^{+12.8}\right)$ & $31.5\left(\left(_{-6.2}^{+4.5}\right)\right.$ \\
\hline \multirow[t]{2}{*}{ (Pyroxene Type) } & $1.5 \mu \mathrm{m}$ & - & - & - & - & $79.4\left({ }_{-31.6}^{+10.0}\right)$ & - & - \\
\hline & $6.0 \mu \mathrm{m}$ & - & - & - & - & - & - & - \\
\hline \multirow[t]{3}{*}{ Forsterite } & $0.1 \mu \mathrm{m}$ & - & - & - & - & $5.8\left({ }_{-0.7}^{+0.9}\right)$ & - & - \\
\hline & $1.5 \mu \mathrm{m}$ & - & - & - & - & - & - & - \\
\hline & $6.0 \mu \mathrm{m}$ & - & - & - & - & - & - & - \\
\hline \multirow[t]{3}{*}{ Enstatite } & $0.1 \mu \mathrm{m}$ & - & - & - & - & - & - & - \\
\hline & $1.5 \mu \mathrm{m}$ & - & - & - & - & - & - & - \\
\hline & $6.0 \mu \mathrm{m}$ & - & - & - & - & - & - & - \\
\hline \multirow[t]{3}{*}{ Silica } & $0.1 \mu \mathrm{m}$ & - & - & - & - & - & - & - \\
\hline & $1.5 \mu \mathrm{m}$ & - & - & - & - & - & - & - \\
\hline & $6.0 \mu \mathrm{m}$ & - & - & $6.4\left(\begin{array}{c}+0.9 \\
-1.1\end{array}\right)$ & $7.1\left(\left(_{-0.5}^{+0.6}\right)\right.$ & - & $8.7\left({ }_{-4.3}^{+4.7}\right)$ & - \\
\hline
\end{tabular}




\section{References}

Adams, F., Lada, C. J., \& Shu, F. H. 1987, ApJ, 312, 788

Apai, D., Pascucci, I., Bouwman, J., et al. 2005, Science, 310, 834

Artymowicz, P., \& Lubow, S. H. 1994, ApJ, 421, 651

Bouwman, J., Lawson, W. A., Dominik, C., et al. 2006, ApJ, 653, 57

Bouwman, J., Henning, Th., Hillenbrand, L. A., et al. 2008, ApJ, 683, 479

Brandeker, A., Jayawardhana, R., \& Najita, J. 2003, AJ, 126, 2009

Broeg, C., Joergens, V., Fernández, M., et al. 2006, A\&A, 450, 1135

Carmona, A., van den Ancker, M. E., Henning, Th., et al. 2008, A\&A, 478, 795

Carpenter, J. M., Mamajek, E. E., Hillenbrand, L. A., \& Meyer, M. R. 2006, ApJ, 651, L49

Chauvin, G., Ménard, F., Fusco, T., et al. 2002, A\&A, 394, 949

Crovisier, J., Leech, K., Bockelee-Morvan, D., et al. 1997, Science, 275, 1904

Damjanov, I., Jayawardhana, R., Scholz, A., et al. 2007, ApJ, 670, 1337

Dorschner, J., Begemann, B., Henning, Th., Jäger, C., \& Mutschke, H. 1995 A\&A, 300, 503

Dullemond, C. P., \& Dominik, C. 2004, A\&A, 421, 1075

Fabian, D., Jäger, C., Henning, Th., Dorschner J., \& Mutschke, H. 2000, A\&A, 364,282

Flaherty, K. M., \& Muzzerole, J. 2008, AJ, 135, 966

Furlan, E., Calvet, N., D’Alessio, P., et al. 2005, ApJ, 628, L65

Gail, H.-P. 1998, A\&A, 332, 1099

Gail, H.-P. 2004, A\&A, 413, 571

Gustafsson, B., Edvardsson, B., Eriksson, K., et al. 2008, A\&A, 486, 951

Hearty, T., Neuhäuser, R., Stelzer, B. et al. 2000, A\&A, 353, 1044

Henning, Th., \& Mutschke, H. 1997, A\&A, 327, 743

Higdon, S. J. U., Devost, D., Higdon, J. L., et al. 2004, PASP, 116, 975

Hogerheijde, M. R., Johnstone, D., Matsuyama, I., et al. 2003, ApJ, 593, L101

Houck, J. R., Roellig, T. L., van Cleve, J., et al. 2004, ApJS, 154, 18

Itoh, Y., Fukuda, N., Nakanishi, N., et al. 2003, ApJ, 586, L141

Jäger, C., Molster, F. J., Dorschner, J., et al. 1998, A\&A, 339, 904

Jayawardhana, R., Wolk, S. J., Barrado y Navascués, D., et al. 2001, ApJ, 550, L197

Jayawardhana, R., Luhman, K. L., D’Alessio, P., \& Stauffer, J. R. 2002, ApJ, 571, L51

Jensen, E. L. N., Mathieu, R. D., \& Fuller, G. A. 1996, ApJ, 458, 312

Juhász, A., Henning, Th., Bouwman, J., et al. 2009, ApJ, in press
Keller, Ch., \& Gail, H.-P. 2004, A\&A, 415, 1177

Kemper, F., Vriend, W. J., \& Tielens, A. G. G. M. 2004, ApJ, 609, 826

Kessler-Silacci, J., Augereau, J.-C., Dullemond, C. P., et al. 2006, ApJ, 639, 275

Kessler-Silacci, J., Dullemond, C. P., Augereau, J.-C., et al. 2007, ApJ, 659, 680

Kurucz, R. L. 1994, Solar abundance model atmospheres for 0, 1, 2, 4,

$8 \mathrm{~km} \mathrm{~s}^{-1}$, CD-ROM No. 19 (Cambridge, Mass.: Smithsonian Astrophysical Observatory)

Lada, C. J., Muench, A. A., Haisch, K. E., Jr., et al. 2000, AJ, 120, 3162

Lahuis, F., \& Boogert, A. 2003, in SFChem 2002: Chemistry as a Diagnostic of

Star Formation, ed. C. L. Curry, \& M. Fich, 335

Luhman, K. L. 2001, ApJ, 560, 287

Luhman, K. L., \& Steeghs, D. 2004, ApJ, 609, 917

Luhman, K. L., Stauffer, J. R., Muench, A. A., et al. 2003, ApJ, 593, 1093

Magnani, L., Blitz, L., \& Mundy, L. 1985, ApJ, 295, 402

Makovitz, D., \& Marleau, F. 2005, PASP, 117, 1113

Malfait, K., Waelkens, C., \& Waters, L. B. F. M. 1998, A\&A, 332, L25

Min, M., Hovenier, J. W., \& de Koter, A. 2005, A\&A, 432, 909

Min, M., Water, L. B. F. M., de Koter, A., et al. 2007, A\&A, 462, 667

Muench, A. A., Lada, E. A., Lada, C. J., \& Alves, J. 2002, ApJ, 573, 366

Pascucci, I., Apai, D., Hardegree-Ullman, E. E., et al. 2008, ApJ, 673, 477

Petr, M. G., Coude Du Foresto, V., Beckwith, S. V. W., et al. 1998, ApJ, 500, 825

Pound, P. W., Bania, T. M., \& Wilson, R. W. 1990, ApJ, 351, 165

Ratzka, Th., Köhler, R., \& Leinert, Ch. 2005, A\&A, 437, 611

Rieke, G., Young, E. T., Engelbracht, C. W., et al. 2004, ApJS, 154, 25

Scott, E. R. D., \& Krot, A. N. 2005, ApJ, 623, 571

Servoin, J. L., \& Piriou, B. 1973, Phys. Stat. Sol., 55, 677

Sicilia-Aguilar, A., Hartmann, L. W., Hérnandez, J., et al. 2005, AJ, 130, 188

Sicilia-Aguilar, A., Hartmann, L. W., Fürész, G., et al. 2006, AJ, 132, 2135

Sicilia-Aguilar, A., Hartmann, L. W., Watson, D., et al. 2007, ApJ, 659, 1637

Sicilia-Aguilar, A., Henning, Th., Juhász, A., et al. 2008, ApJ, 687, 1145

Scholz, A., Jayawardhana, R., Wood, K., et al. 2007, ApJ, 660, 1517

Swain, M. R., Bouwman, J., Akeson, R. L., Lawler, S., \& Beichman, C. A. 2008, ApJ, 674, 482

van Boekel, R., Waters, L. B. F. M., Dominik, C., et al. 2003, A\&A, 400, L21

White, R. J., \& Basri, G. 2003, ApJ, 582, 1109

Werner, M. W., Roellig, T., Low, F., et al. 2004, ApJS, 154, 1

Zimmermann, T., \& Ungerechts, H. 1990, A\&A, 238, 337 